\title{
Rapid Prediction of Installed Jet Noise From RANS
}

\author{
JAMES BRIDGES \\ Acoustics Branch, NASA Glenn Research Center \\ 08 June 2019
}

\begin{abstract}
A new method of computing jet noise, called $m S r c$, was developed on the general principles of acoustic analogies. In the method, the problem of translating turbulent flow energy into acoustic energy at a far-field observer is broken into two parts, a calculation of acoustic source strengths and then their propagation. The acoustic sources are related to turbulent quantities in the jet plume in a robust manner. The propagation, which is more properly computed using a Green's function accounting for nonuniform speed of sound and solid surfaces, is instead modeled using commonly observed features of jet acoustic far field directivity, and by diffraction barrier theory for surfaces. The $\mathrm{mSrc}$ method does not require gradients of the predicted flow field, allowing it to make direct use of unstructured RANS CFD methods, including embedded boundary codes. Such codes do not require specification of surface meshes, and auto-refine their grid to resolve flow gradients, putting resolution where it is required without $a$ priori user input. Being able to use radically unstructured flow input makes $m S r c$ an efficient method of estimating noise of complex nozzles installed on aircraft. Many validation cases are presented to demonstrate the accuracy and range of applicability of the $m S r c$ method for representative jet noise applications.
\end{abstract}

\section{Introduction and motivation}

Jet noise has been notoriously difficult to predict. Methods for prediction run from purely empirical to exquisitely detailed solutions of fundamental physics of fluid flow. Empirical methods are usually built around databases of geometrically idealized experiments[1]. They give predictions of far-field noise within seconds from limited user input, usually only a few one-dimensional parameters describing the nozzle and flow conditions. However, real-world aspects of exhaust systems, including duct bifurcations, pylons, presence of wings or fuselage, angle of attack, incomplete mixing of internal mixers, swirl, centerbodies, and including various mixing enhancement features designed to reduce the noise are not captured in general empirical codes. These aspects are often addressed in individual databases and empirical corrections. The biggest problem with empirical approaches is that they offer little insight into how changes in geometry can affect noise, something required to truly design low-noise exhaust systems.

On the other extreme are methods, such as large-eddy simulations (LES), that compute the details of the jet flow down to the smallest swirl that impacts noise. These methods are becoming feasible for real-world problems, but require months for a single solution. Designers cannot wait this long for answers. These high-fidelity methods do allow great insight into noise generation, and are invaluable for this task. They are also the only method of reliably predicting flow resonance problems.

In between these two prediction methods lie acoustic analogy approaches [2,3,4]. These start by manipulating the Navier-Stokes equations into wave equations, preserving terms that are normally ignored in linear acoustics. Only when these terms have to be quantified by high-order turbulent statistics are approximations and models introduced. The most popular acoustic analogy methods use Reynolds-Averaged Navier-Stokes (RANS) flow predictions as input for the source terms, but it is the calculation of the Green's function that couples the source terms to the far-field observer that is the most problematic. This calculation, particularly in cases where the inhomogeneous field lacks symmetries, such as non-round jets [5], can be computationally prohibitive. Methods for computing the Green's function are also hobbled by the need for first-order or higher spatial derivatives of the velocity and temperature field of the jet, which can be a practical problem when using output from CFD codes. CFD codes that are high-order and use structured grids usually generate smooth derivatives, but such codes are slow to use on practical nozzle geometries, primarily because gridding is so difficult. Unstructured grid CFD codes are much easier and faster to use, but it is

\footnotetext{
$\dagger^{\dagger}$ Acoustics Branch; AIAA Associate Fellow
} 
difficult to produce interpolated fields with adequate smoothness for the Green's function solver. Acoustic analogy methods, including producing the CFD required for their propagation phase, typically require days for a prediction.

Exhaust system design, especially at the conceptual phase, requires fast, and robust prediction of jet noise, especially if one wants to use geometric features, including installation, to reduce the impact on ground observers. The prediction method should also give insight into how the noise predicted was generated in a manner that can be addressed by design. For steady turbulent flows, this means showing what regions of the turbulent flow produced what frequency of noise in what direction. This can then be tied back through the computed flow to geometric parameters that the designer can modify. This is also the requirement for optimization approaches that need to use partial derivatives of each step in the chain from geometry to flow to noise to be able to minimize noise by manipulating geometry.

A new method has been developed that is loosely based on acoustic analogies of the past, but circumvents several difficulties, especially with Green's functions, to arrive at a code that does not require computation of flow gradients from the CFD output. This simplification has tradeoffs, but allows for a quick turnaround, and promises to be of use in optimization schemes. In a nutshell, it uses a simplified source model, very much like those used in acoustic analogy methods, but then uses models for the coupling of these source to the far-field instead of computing a true Green's function. A key aspect is the method's ability to use unstructured grid CFD, including fast imbedded-boundary codes, because it does not require flow gradients.

The exposition begins by illustrating the commonly understood distinction in jet noise between noise from smallscale sources and large-scale sources. Much has been made of this distinction, leading to approaches that only capture the large-scale source. In military applications where the large-scale noise is so dominant, this makes sense. But for commercial applications the exhaust noise at broadside angles is significant at frequencies where humans are sensitive and must be predicted accurately.

Figure 1 shows spectral directivity from a typical subsonic jet, in this case a single stream jet with acoustic Mach number $M a=0.9$ and static temperature $T s R=2.27$, documented in [6]. From the two views of the surface one sees a surface shaped like a parabola in frequency, slightly sloping from small angles (forward) and increasing going aft. At roughly $150^{\circ}$ the directivity has the shape of a different parabola in frequency, narrower with a lower frequency peak. This parabola is much higher amplitude than the one at forward angles. Figure $1 \mathrm{~b}$ is an attempt to quantify these visual trends by using two shapes, called small-scale and large-scale, which when added together nearly replicate the original data.

A method for predicting these two shapes for a generic jet, called ' $m S r c$ ', will be described in this paper. Following that, the method will be validated for a variety of jets, including installed configurations. Validation not only includes comparisons of far-field sound with measurements, but also of the CFD and source distributions. The validation exercises cover a wide range of jet flows to show where the $\mathrm{mSrc}$ method works, as well as where it should be used with caution. 


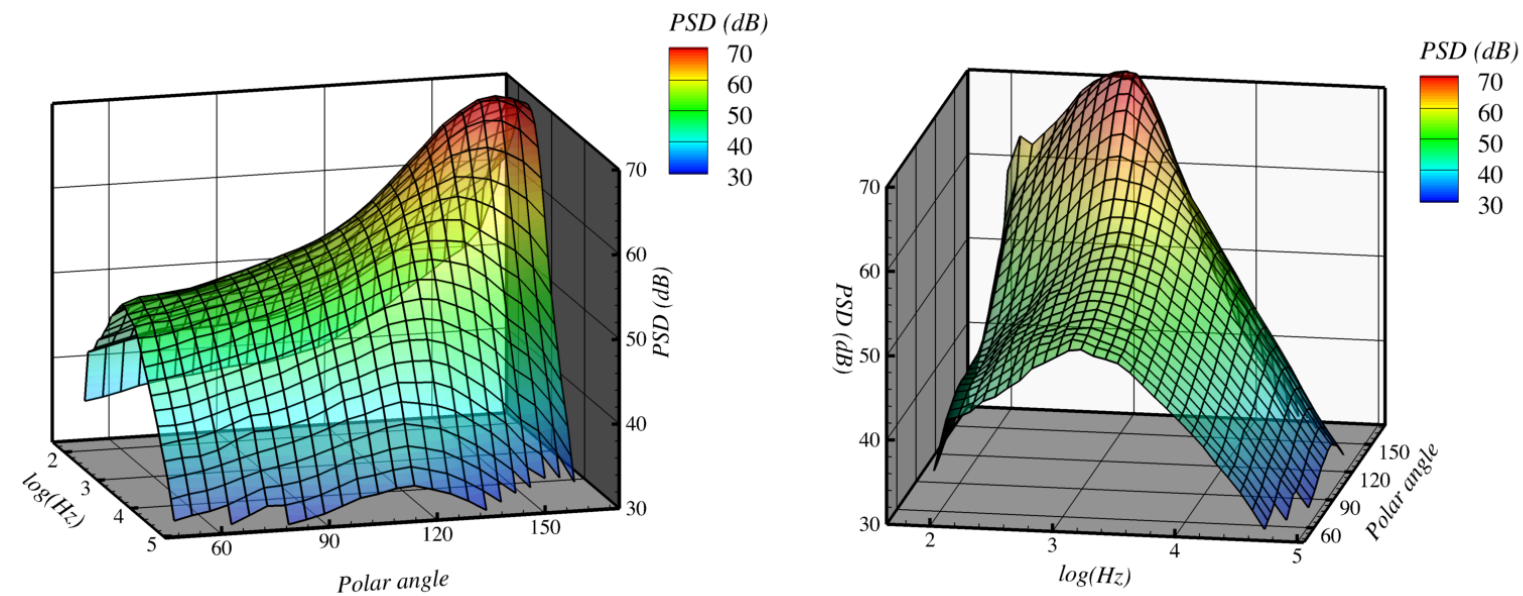

(a)
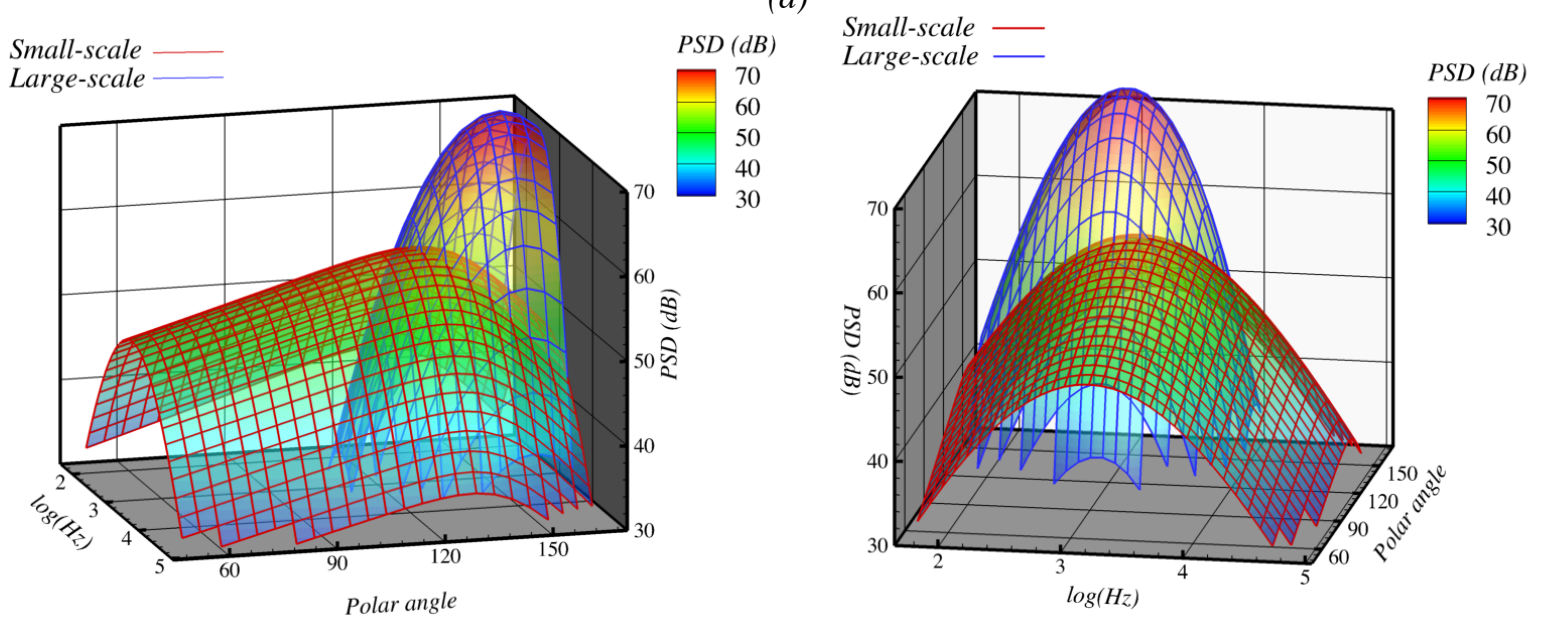

(b)

Figure 1. Visual depiction of spectral directivity of far-field noise. (a) Measured power spectral density (PSD), (b) modeled small-scale and large-scale components of far-field noise.

\section{II.Small-scale sound}

Starting with the small-scale source model, the essence of this model comes from earlier acoustic analogy methods [4] which established the basic scaling of noise sources with TKE and the need to separately consider sources associated with turbulent momentum fluctuations and with turbulent enthalpy fluctuations.

\section{A. Small-scale source density $S S$}

The small-scale sources $S S_{n}(f)$ are given by

$$
\begin{aligned}
& S S_{n}(f)=S S_{m_{n}}(f)+S S_{e_{n}}(f), \\
& S S_{m_{n}}(f)=C_{s s m}\left(\frac{\rho_{n}}{\rho_{\infty}}\right)^{2} \kappa_{n}^{7 / 2} 10^{\left(-A_{s s m}\left(\ln \left(B_{s s m} \frac{\varepsilon_{n} / \kappa_{n}}{f}\right)\right)^{2}\right)} V_{n} \\
& S S_{e_{n}}(f)=C_{s s e}\left|\frac{\rho_{n}}{\rho_{\infty}}-1\right|^{2} \kappa_{n}^{5 / 2} 10^{\left(-A_{\text {sse }}\left(\ln \left(B_{s s e}\left(\frac{\rho_{n}}{\rho_{\infty}}\right)^{1 / 2} \frac{\varepsilon_{n} / \kappa_{n}}{f}\right)\right)^{2}\right)} V_{n}
\end{aligned}
$$

where

$\rho \quad$ is density

$\kappa \quad$ is turbulent kinetic energy

$\varepsilon \quad$ is dissipation 
$V \quad$ is volume of cell

$f \quad$ is frequency at observer

$A \quad$ is source spectral width coefficient

$B \quad$ is source spectral center adjustment

$\mathrm{C}$ is the overall scaling coefficient

$n \quad$ is the index of the $\mathrm{n}^{\text {th }}$ element of the computational domain.

Note that there is no dependence on observer or source location in equation (1). Directivity will be addressed separately in the next section. At its heart, $m S r c$ is the kernel of an acoustic analogy without the Green's function that specifies how the source is coupled to the acoustic far-field, and without models for source directivity due to turbulence anisotropy. There are no factors in the spectral shape other than the CFD input, which is totally local with no global information about the flow, such as jet diameter. The Green's function for a source in a jet at $90^{\circ}$ is unity, so in a sense this is a prediction of jet mixing noise at polar angle $90^{\circ}$. To get the sound at an observer at right angles to the jet we sum the contributions from all the cells in the computational domain, and apply a global coefficient to make the sound match data at a given radius from the jet.

\section{B. Small-scale directivity $D S$}

The model for directivity was derived empirically from the SMC000 nozzle data over the Tanna matrix of flows [7,8]. This dataset covers single jet plumes with acoustic Mach numbers $M a$ ranging from 0.5 to 1.5, and static temperature ratios $T_{S} R$ ranging from less than 1 (unheated) to 2.7. For discussion, define the polar directivity $\Delta_{\mathrm{s}}(\phi)$ to be the $P S D$ at a particular frequency $f^{*}$ normalized by its value at $90^{\circ}$ :

$$
\Delta_{\mathrm{s}}(\phi)=P S D\left(f^{*}, \phi\right) / P S D\left(f^{*}, 90^{\circ}\right) \text {. }
$$

The subscript $\mathrm{S}$ denotes that this directivity is used for the directivity of small-scale (high frequency) sound. The directivity of the measured jet noise at broadside polar angles $\phi<110^{\circ}$, when given by the ratio of $P S D$ in $\mathrm{dB}$, is roughly linear with polar angle. If one only considers frequencies above the spectral peak, $\Delta_{\mathrm{s}}(\phi)$ takes a quick rolloff with polar angle after roughly $130^{\circ}-145^{\circ}$. Presumably, this is due to refraction of the high frequency sources near the nozzle as they propagate through the jet plume. Rather than compute this Green's function directly, a model, $D S$, was created that matches the behavior of the experimental $\Delta_{\mathrm{s}}(\phi)$ data over the range of $M a$ and $T s R$ included in the Tanna matrix. For $\phi<110^{\circ}, \Delta_{\mathrm{s}}(\phi)$ is observed to have a slope that varies primarily with $M a$, given by

$$
0.06 * M a+0.058 \text {. }
$$

When $\Delta_{\mathrm{s}}(\phi)$ is given as a ratio of sound power (not $\mathrm{dB}$ ), this portion of the small-scale directivity becomes

$$
\operatorname{SSlope}(\phi ; M a)=10^{(0.006 * M a+0.0058) *(\phi-90)},
$$

where 90 assumes that the directivity is anchored at $\phi=90^{\circ}$.

The aft angle roll-off of the small-scale sound field $\Delta_{\mathrm{s}}(\phi)$, when expressed in sound power, is well fitted by $\tanh (\phi)$.

$$
\operatorname{SFall}(\phi)=0.5 *\left(1-\tanh \left(\frac{\phi-\phi_{s 0}}{\phi_{S 1}}\right)\right),
$$

where $\phi_{s 0}$ is the polar angle where the power is cut in half, and $\phi_{s 1}$ is a width parameter describing how quickly the sound power rolls off with $\phi$. Models for these parameters will be coordinated with the model for directivity of the large-scale source, covered in the next section.

Combining the slope and rolloff models gives the directivity for the small-scale sources, to be multiplied by the small-scale noise $S S$ (in power, not $\mathrm{dB}$ ) at $\phi=90^{\circ}$. This small-scale noise is from both momentum $S S_{m}$ and enthalpy $S S_{e}$ terms.

$$
D S(\phi ; M a)=\operatorname{SSlope}(\phi ; M a) * \operatorname{SFall}(\phi)
$$

A visual check of this model is given in Figure 2, showing the model $D S$ against $\Delta_{\mathrm{s}}(\phi)$ data at the high frequency $S t=10$ from unheated jets at $\mathrm{Ma}=0.5$ and 0.9 . Note that this model requires an external input of $M a$ for the jet flow, a restriction to the otherwise-local computation by $m S r c$. 


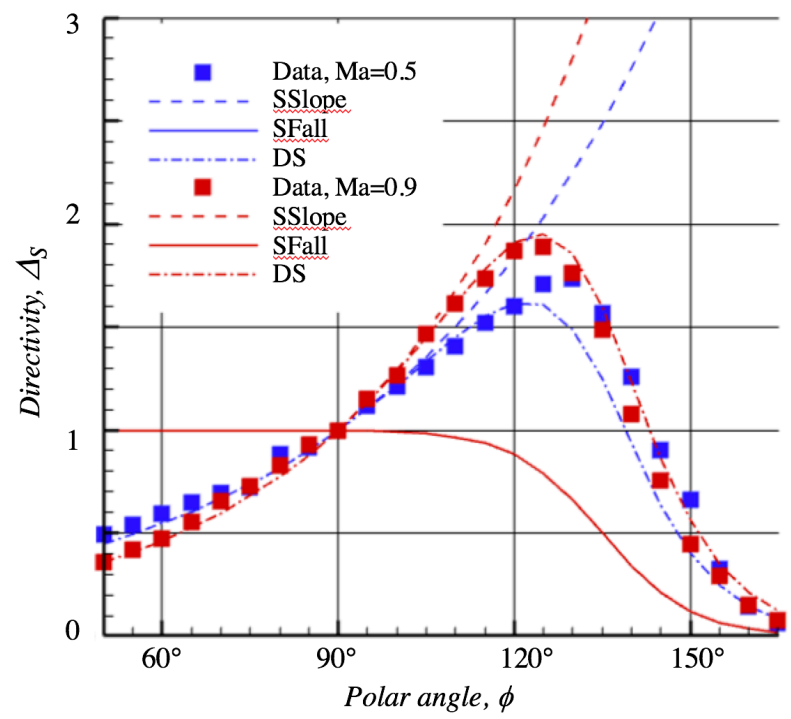

Figure 2. Small-scale directivity DS, combining both SSlope (dashed lines) and SFall (solid line) functions. The combined directivities are compared with $\Delta_{\mathrm{s}}$ data at $S t=10$ for two jet unheated jet conditions, $M a=0.5$ and 0.9 .

The small-scale sound field $S S(f)$ is therefore computed by summing the contributions of the small-scale sources $S S_{n}(f)$ from each cell of the computation domain over the CFD volume, and then multiplying by the small-scale directivity $D S(\phi, M a)$ :

$$
S S(\phi, f)=D S(\phi, M a) * \sum_{n} S S_{n}(f) .
$$

\section{Large-scale sound}

Next, we model the large-scale noise, by which we mean the low-frequency, aft peak that dominates the spectral directivity. First, some motivation. From LES of jets, the acoustic waves strongly radiating to the aft angle seem to originate at the end of the potential core of jets. In addition, various decompositional approaches have been applied to the large-scale noise, which indicate they arise from the coupling of low-order spatial modes of the turbulent energy to the acoustic field. This coupling seems to require that the lengthscale of the turbulence match that of the low-order azimuthal modes. Observing that turbulence lengthscales generally match the jet diameter around and aft of the potential core, it is proposed that the TKE which has lengthscales matching the jet diameter is what feeds the largescale noise. Using a spatial filter on the turbulence will select contributions only from regions where this match occurs.

\section{A. Large-scale source density $S L$}

The large-scale source is modeled on much the same functional form as the small-scale sources, but with different scaling in TKE and includes a spatial filter to only include contributions from the turbulence whose lengthscale matches the equivalent jet diameter. The directivity of this source is very significant, having almost no energy at $90^{\circ}$. The source model was calibrated primarily by matching the aft peak of the jet noise, both in polar directivity and spectral shape. Empirically, once the small-scale sound (source+directivity) is subtracted from the full spectral directivity, what's left is considered the large-scale noise. This is not a first-principles derivation; it comes from observation of jet data. It will only be a viable approach if the CFD-related aspects of the source modeling, such as prediction of turbulent lengthscales, captures the noise impacts of nozzle geometry change.

The spatial filtering that determines what turbulent energy couples to the aft acoustic field is done using a lengthscale filter to pick out $T K E$ that has lengthscales commensurate with the low-order modes of the jet. These loworder modes have scales on the order of the jet diameter, so the jet diameter is the scaling factor used in the lengthscale filter. The functional form of the lengthscale filter $\Psi$ is

$$
\Psi(\text { Djet })=10\left(-\left(\ln \left(\frac{\kappa^{3 / 2} / \varepsilon}{\text { Djet }}\right)\right)^{2}\right)
$$

where $\kappa^{3 / 2} / \varepsilon$ produces a turbulence lengthscale to be compared with the reference jet diameter Djet. 
Looking at RANS solutions (Figure 3) shows that such a filter does peak at regions just downstream of the potential core. In the plot, color contours show the filter while black contour lines show the mean velocity field for an unheated, $M a=0.9$ jet.

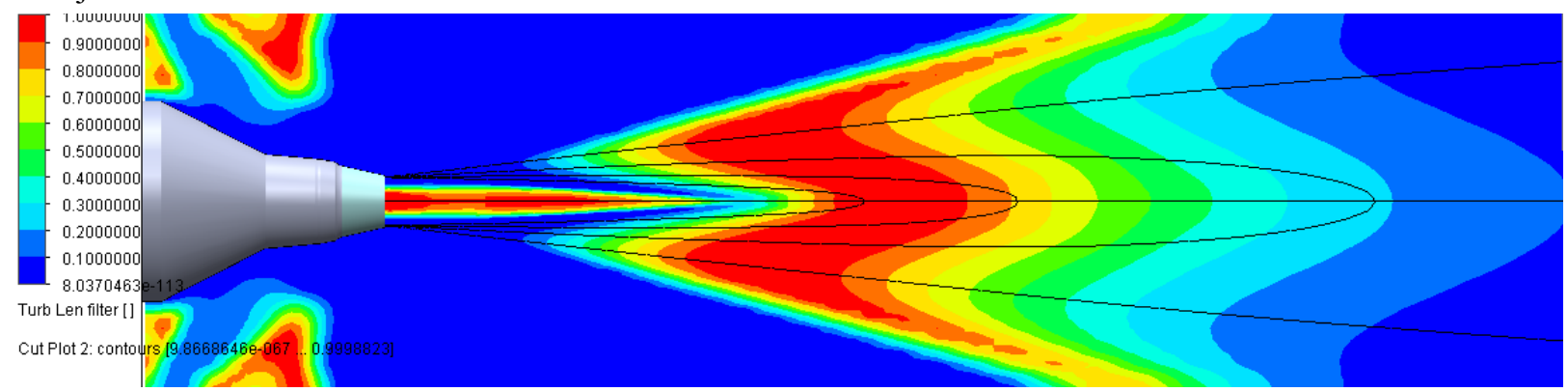

Figure 3. Contour map of lengthscale filter $\Psi$ with isolines of TKE for visual reference.

The spectral shape at the peak aft angle for jets in the Tanna matrix is similar to the spectral shape of the smallscale source, but has a lower peak frequency and narrower shape. Applying the same functional form as the smallscale source, but with different $T K E$ exponent gives a spectral shape that matches the peak spectrum. The coefficients used to shift the peak frequency and spectral width are nearly unity, giving confidence that the model has some basis in physics.

Picking off peak amplitudes of the cold jets in the Tanna matrix, it was noted (again from empirical observation) that the peak amplitude scales not as $\mathrm{TKE}^{7 / 2}$, but as $\mathrm{TKE}^{9 / 2}$. A two-source model was developed for momentum terms and enthalpy terms, mirroring the small-scale mixing source. Unlike the small-scale source model, the large-scale source model, through the spatial filtering, requires a global parameter, jet diameter Djet, which the user supplies external to the local CFD values.

In the efficient volumetric form of the $m S r c$ approach, the contribution of each flow volume $n$ in the RANS solution to the large-scale source strength is given by

$$
\begin{aligned}
& S L_{n}(f)=S L_{m_{n}}(f)+S L_{e_{n}}(f) \text {, } \\
& S L_{m_{n}}(f ; \text { Djet })=C_{s l m}\left(\frac{\rho_{n}}{\rho_{\infty}}\right) \kappa_{n}^{9 / 2} 10^{\left(-A_{s l m}\left(\ln \left(B_{s l} \mathrm{~m} \frac{\varepsilon_{n} / \kappa_{n}}{f}\right)\right)^{2}\right)}{ }_{10}\left(-\left(\ln \left(D_{s l m}\left(\frac{\rho_{n}}{\rho_{\infty}}\right) \frac{\kappa^{3 / 2} / \varepsilon}{D j e t}\right)\right)^{2}\right) V_{n} \text {, } \\
& S L_{e_{n}}(f ; \text { Djet })=C_{\text {sle }}\left|\frac{\rho_{n}}{\rho_{\infty}}-1\right|^{2} \kappa_{n}^{7 / 2} 10^{\left(-A_{\text {sle }}\left(\ln \left(B_{\text {sle }} \frac{\varepsilon_{n} / \kappa_{n}}{f}\right)\right)^{2}\right)}{ }_{10}\left(-\left(\ln \left(D_{\text {sle }}\left(\frac{\rho_{n}}{\rho_{\infty}}\right) \frac{\kappa^{3 / 2} / \varepsilon}{D j e t}\right)\right)^{2}\right) V_{n}
\end{aligned}
$$

where

$\rho \quad$ is density

$\kappa \quad$ is turbulent kinetic energy

$\varepsilon \quad$ is dissipation

$V \quad$ is volume of cell

$f \quad$ is frequency at observer

$A \quad$ is source spectral width coefficient $\left(A_{s l m}=A_{s l e}=1.0\right)$

$B \quad$ is source spectral center adjustment $\left(B_{s l m}=B_{s l e}=1.1\right)$

$\mathrm{C} \quad$ is the overall scaling coefficient $\left(C_{s l m}=0.0002, C_{s l e}=7\right.$. $)$

$\mathrm{D} \quad$ is the lengthscale filter width coefficient $\left(D_{\text {slm }}=D_{\text {sle }}=0.8\right)$

$n \quad$ is the index of the $\mathrm{n}^{\text {th }}$ element of the computational domain.

Djet is the reference jet diameter

This source density is integrated over all the cells in the RANS solution, although the lengthscale filter effectively keeps most regions of the flow from contributing to the large-scale source. 


\section{Large-scale directivity $D L$}

As stated above, the directivity of the large-scale source is its most distinguishing feature, especially when viewed in sound power instead of dB. Figure 4 shows the directivity of the far-field jet sound, $\Delta_{\mathrm{L}}(\phi)$, which differs from $\Delta_{\mathrm{s}}(\phi)$ by being normalized by the value at the aft peak polar angle. The plot contains curves covering a wide range of $M a$ and $T s R$, but with the exception of the unheated jets (solid lines) the directivities are very similar in this view. This is the directivity $\mathrm{mSrc}$ seeks to model and to apply to the large-scale source.

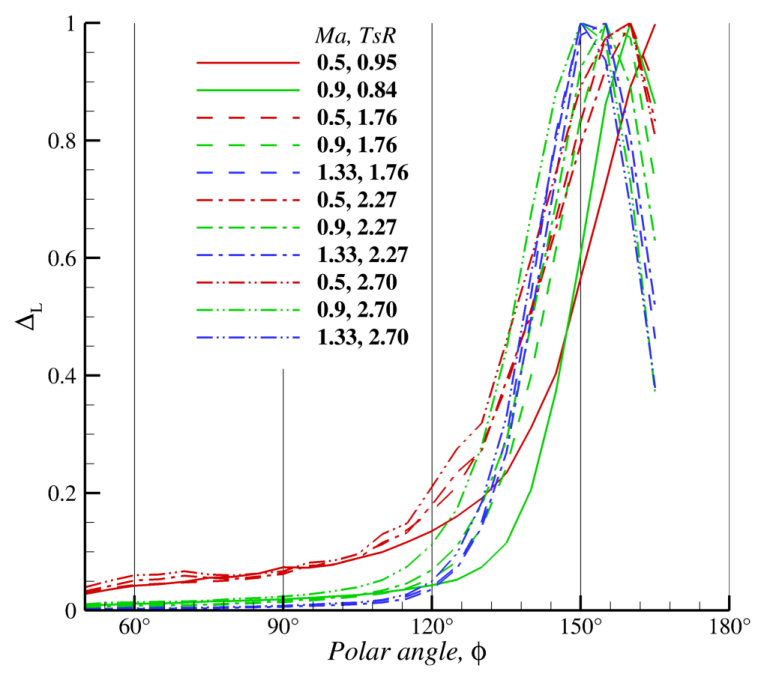

Figure 4. Normalized directivities at peak frequencies for several single-stream jets in Tanna matrix. Ordinate is linear in sound power (not $\mathbf{d B}$ ).

Everyone's favorite functional form, the Gaussian, is brought out once again, and found to do a good job of fitting the directivity of the large-scale sound with appropriate choice of parameters. In addition, it will be shown that the small-scale directivity can be added to the Gaussian to match the total sound directivity seamlessly, validating the addition of these two sources if the source strengths are properly matched.

The large-scale directivity $D L$ is

$$
D L(\phi)=e^{-\left(\left(\left(\phi-\phi_{l 0}\right) / \phi_{l 1}\right)^{2}\right)} .
$$

The coefficients $\phi_{10}$ and $\phi_{11}$ are empirically derived functions of global reference values for $M a$ and $T_{s} R$. In the $m S r c$ code, a global reference value for the $M a$ is supplied by the user. In practice it seems to be best chosen as the peak velocity divided by ambient speed of sound. The reference value for $T s R$ is obtained internally by a TKEweighted integral (carried out as summations over CFD cells) of the fluid density to find the 'average' density associated with the TKE. This pulls out the relevant density because the TKE is highly correlated with the source spatially. Empirical relations serve to convert this measure of density into static temperature ratio of a single-stream jet (essentially ignoring static pressure variations in the plume). This estimate of an equivalent static temperature ratio is the basis for the empirical $D L$ directivity factor.

$$
T s R_{\text {ref }}=1+6\left(\rho_{\infty} \frac{\sum_{n} \kappa_{n} V_{n}}{\sum_{n} \rho_{n} \kappa_{n} V_{n}}-1\right) .
$$

The peak polar angle $\phi_{10}$, in degrees, was derived empirically to be

$$
\phi_{l 0}=-11(M a-1)-4\left(T s R_{r e f}-1\right)+158 .
$$

The width of the polar peak could be made to be a function of $M a$ and $T s R$ as well, but for now are simply taken to be a constant width of $15^{\circ}$.

As described above, the rolloff of the small-scale directivity $D S$ is coordinated with the peak location of the largescale to smooth over where they overlap. Specifically,

$$
\phi_{s 0}=\phi_{l 0}-\phi_{l 1} \text {, and } \phi_{s 1}=\phi_{l 1} \text {. }
$$

The end result of these models for directivity is shown in Figure 5. The Gaussian model for the large-scale directivity (Figure 5) of the peak frequency for a hot subsonic jet is very good except for between $120^{\circ}$ and $130^{\circ}$ where 
it slightly underpredicts the amplitude. This deficit is somewhat filled when the large-scale directivity is combined with the small-scale directivity, shown as an angled straight line in $\mathrm{dB}$ scale (Figure 6). The total directivity still somewhat underpredicts the data at the cross-over angle of $120^{\circ}$, a deficit that will be noted in the validation exercises and is a direct result of using such simple functional forms for $D S$ and $D L$.

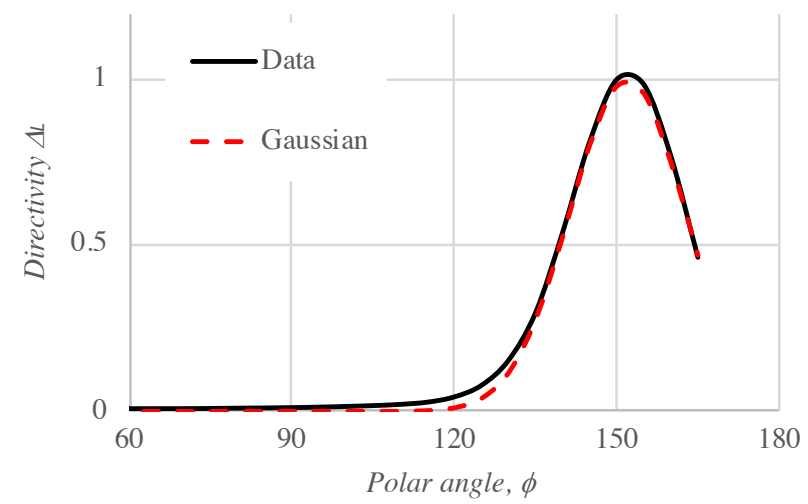

Figure 5. Model of large-scale source directivity against normalized data at $\mathrm{St}=\mathbf{0 . 3}$.

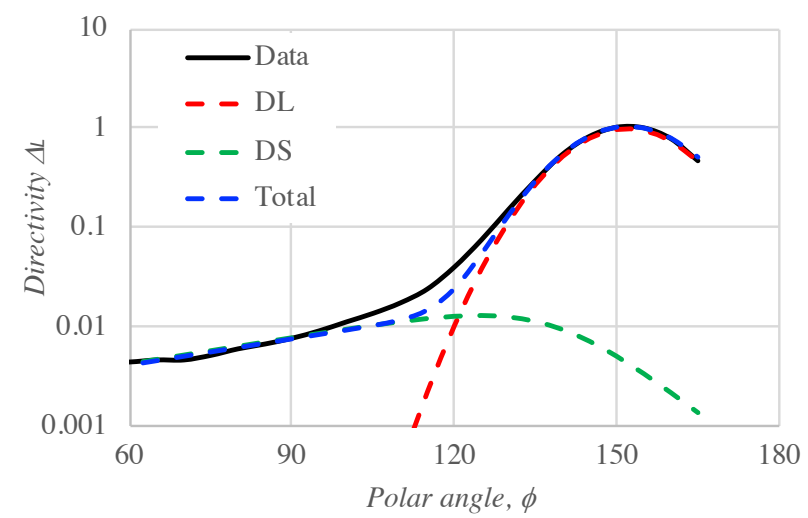

Figure 6. Combined models of small-scale and large-scale directivities and their total (in $\mathrm{dB}$ ), compared with normalized data at $\mathrm{St}=\mathbf{0 . 3}$.

The spectral directivity of the large-scale sound is the product of the source density $S L_{\mathrm{n}}(f, D j e t)$ and the directivity $D L(\phi, M a, T s r)$.

$$
S L(\phi, f ; M a, D j e t, T s R)=D L(\phi ; M a, T s R) * \sum_{n} S L_{n}(f ; D j e t) .
$$

With all the dependencies made explicit, it is clear that the calculation of the large-scale acoustic field requires some parameters beyond just the local values of the turbulent field, specifically Djet and Ma. This somewhat limits the generality of the method, but it serves to emphasize how the peak aft jet noise is a property of the overall geometry, not a general property of generic turbulent flow. Otherwise, the method only uses density, TKE, and dissipation from the CFD.

\section{Solid surfaces}

Computing the Green's function for a jet flow is hard enough without surfaces, but it is the impact of surfaces which is of interest in many advanced aircraft designs. We have so far avoided actually computing any Green's functions, due to our aversion to computing spatial derivatives. Therefore, it is tempting to use other methods to estimate the acoustic impact of a surface on the noise of a jet installed on an aircraft. One good choice would be the Kirchhoff integral method [9]. The referenced paper clearly demonstrates the superiority of this shielding method to that which is demonstrated here. However, at this time a much simpler approach is taken.

To apply a simplified shielding model, first make the assumption that the sources are independent of the surface in their acoustic coupling, and treat the interaction of their sound with nearby surfaces as a simple acoustic propagation problem, i.e. one of shielding or reflection. This is not to say that the impact of the surface on the turbulent flow is being ignored - that effect is accounted for in the CFD of the installed configuration! Introduce an attenuation factor 
$D H_{n}(\boldsymbol{x}, f, \phi)$ to describe the modification of the sound received by an observer at $\phi$ from a source at the $n^{\text {th }}$ cell, location $\boldsymbol{x}$, of the numerical domain. This attenuation factor could be a simple view factor (you don't hear what you don't see), it could come from a full linearized Euler calculation (tailored Green's function [10-12]), or from something in between, such as the Kirchhoff integral method. For demonstration purposes and pure computational speed, we will consider, Maekawa's barrier theory [13]. The latter is documented in the WING module of ANOPP, and the method has been implemented in $m S r c$ based on descriptions of WING in the ANOPP technical documentation. The sound spectra at the far-field observer is given by applying the directivity $D H_{n}$ for the surface relative to the source and observer to each $n^{\text {th }}$ source element as it is summed. So, the small-scale sound SS is given by the summation of the source strengths $S S_{n}$ multiplied by the surface-created directivity $D H_{n}$, and the sum multiplied by small-scale directivity $D S$, e.g.,

$$
S S(\boldsymbol{x}, f, \phi)=D S(\phi) * \sum_{n} S S_{n}(f, \boldsymbol{x}) D H_{n}(\boldsymbol{x}, f, \phi) .
$$

A similar expression is used for the large-scale sound SL, substituting $D L$ for $D S$ and $S L_{n}$ for $S S_{n}$. Note that this approach cannot capture the creation of noise by the scattering of the source by the surface trailing edge. In essence, this is an approximation only valid when the source is far away from the surface and in a homogeneous medium, all of which is rarely true in installed jets. However, it will be used as a first approximation to the impact of installation, with validation cases to show where its results should not be believed. More rigorous methods of computing the shielding/reflection of a surface for sources computing using $m S r c$ are being explored. In the meantime, the Maekawa method is being used for conceptual design activities for commercial supersonic aircraft. [14]

\section{Putting the models together}

The main utility of the $\mathrm{mSrc}$ prediction is to predict the impact of modifications to the mixing noise by threedimensional nozzle modifications or by shielding from installations. That is, while the prediction of absolute jet mixing noise by the $m S r c$ method is imperfect, the differences in the mixing noise from modifications to the nozzle are often predicted quite well. Note that in Equations $1-5$ above, the parameters required from the RANS CFD are density, TKE, and dissipation. There are no spatial derivatives of the mean velocity; in fact, the mean velocity is not an input to the method. This gives great flexibility in what RANS code and method is used, and makes $m S r c$ very robust.

The full prediction of three-dimensional source density PSD3 from all sources, with propagation and shielding effects, is made by

$P S D 3(x, y, z, f, \phi)=D S(\phi) \sum_{n} S S_{n}(x, y, z, f) D H_{n}(x, y, z, f, \phi)+D L(\phi) \sum_{n} S L_{n}(x, y, z, f) D H_{n}(x, y, z, f, \phi)$

Integration of this three-dimensional source density over successive directions yields PSD2 (a pseudo-phased array view), PSD1 (axial distribution of source strength), and finally PSD (far-field spectral directivity).

$$
\begin{aligned}
P S D 2(x, y, f, \phi) & =\int \operatorname{PSD} 3(x, y, z, f, \phi) d z \\
\operatorname{PSD} 1(x, f, \phi) & =\int \operatorname{PSD} 2(x, y, f, \phi) d y \\
\operatorname{PSD}(f, \phi) & =\int \operatorname{PSD} 1(x, f, \phi) d x
\end{aligned}
$$

A fruitful application of the $m S r c$ code is to look at the distribution of mixing noise sources in a jet. This is especially important if one is trying to modify the flow to maximize the impact of shielding. It is also a good way to check the prediction at an intermediate level by comparing PSD2 results against phased array measurements. Furthermore, if one wishes to construct a one-dimensional model of source distribution suitable for simple empirical models of shielding, then the integration is carried out over both cross-stream dimensions, producing a map of source strength as a function of axial location (PSD1). Other intermediary results, such as the shielding function $D H(\boldsymbol{x}, f, \boldsymbol{\phi})$, or the source density of the individual components, $S S$ and $S L$, can give engineering insight.

\section{Validating $m S r c$}

Unlike acoustic analogy methods derived from first-principles, $m S r c$ makes its models at a much higher level and should be expected to have less 'physics' built in. Hence, it is critical to validate the results carefully to understand where the modeling fails and results are not to be trusted. Validation has been done on a series of increasingly complicated jet flows to establish the range of validity of the $\mathrm{mSrc}$ method. Note that $\mathrm{mSrc}$ does not predict the noise from broadband shock noise and screech, nor from the hydrodynamic interaction with solid edges, such as in turbulent 
flow over a plate ('trailing edge noise problem'). It should be able to predict the noise of jets in the following configurations, which will be demonstrated in this paper:

- Single-stream jets of various temperatures,

- Dual-stream coaxial jets with heat,

- Single-stream jets from nozzles with enhanced mixing features.

- Jets in some proximity to surfaces (excluding the edge-induced noise).

Fortunately, the author has access to many flow and noise datasets that include these features, and could apply RANS CFD and $m S r c$ to these problems. The predictions were checked against previously documented experimental results. RANS CFD results were compared to PIV data, source distributions were compared against phased array measurements, and far-field spectra were compared against microphone data acquired in the geometric far-field. When the far-field acoustic predictions missed the experimental data, each step in the prediction chain, from CFD to integration of sources, were examined to find the reason for the failure. Insights into the physics of jet noise were also obtained during this examination. Highlights from this validation effort are given in the remainder of the paper.

\section{A. Single-stream, round jets}

Single-stream jet flows have been studied extensively using the SMC000 nozzle on the NASA Small Hot Jet Acoustic Rig (SHJAR), measuring flow with particle image velocimetry (PIV) [15-20], sound source distributions with phased arrays, and far-field sound with high-quality microphones. For many test campaigns, measurements were made over a matrix of velocities and temperature ratios first laid out in an AFRL report [7] and AIAA papers attributed to Tanna, hereafter referred to as the 'Tanna matrix'. To cover a wider range of supersonic flows, several single-stream jets were included using nozzles designed by method of characteristics [19] to be shock-free and hence not having broadband shock or screech.

\section{CFD predictions vs PIV data}

Two RANS codes have been used for input to $m S r c$, FUN3D [21] and Flow Simulation by Mentor Graphics [22]. The former uses a body-fitted, unstructured grid and is run with the k-omega/SST turbulence model. The latter (referred to as SolidWorks Flow Simulation, or 'SWFS') is an embedded boundary method code that is licensed through Dassault Design Systems and tightly integrated with their SolidWorks ${ }^{\mathrm{TM}}$ CAD software. Both codes have been benchmarked against PIV data acquired in a series of single-stream jets, and best practices developed for grid generation, refinement strategies, etc. The SWFS code being used primarily in the current work, is generally run with standard default settings, these being found to produce a satisfactory answer in most cases within minutes to hours. More importantly, although the codes do not precisely replicate the experimental validation data, they seem to produce the same answer which is as good as any other RANS codes and turbulence models that have been tested. SWFS was the CFD code used for the majority of the validation cases presented here.

A common measure for accuracy of CFD codes on jet flows, at least acoustically speaking, is how well they predict potential core length and turbulence levels. Figure 7 shows centerline and lipline mean velocity and TKE profiles for a hot subsonic jet validation case available through the Langley Turbulence Model Resource website. The website not only has the NASA consensus experimental data, but also solutions generated by the WIND RANS code using two different turbulence models. Consistent with most RANS codes, the potential core is predicted to be longer than is commonly measured, and the location of the peak TKE on the centerline is similarly shifted downstream. Here the peak of the TKE on the jet centerline by $m S r c$ is roughly $20 \%$ further downstream than was measured by PIV . However, the peak amplitude of the TKE is correct at both centerline and lipline, important for predictions of noise. Predictions at other flow conditions in the Tanna matrix for a single round jet had similar degrees of accuracy. The main distinction between the solutions shown in the figure is that the WIND solution were run on a structured axisymmetric grid for hours on a supercomputer cluster, while the SWFS solution ran on an auto-refined imbedded boundary grid for minutes on a laptop. 


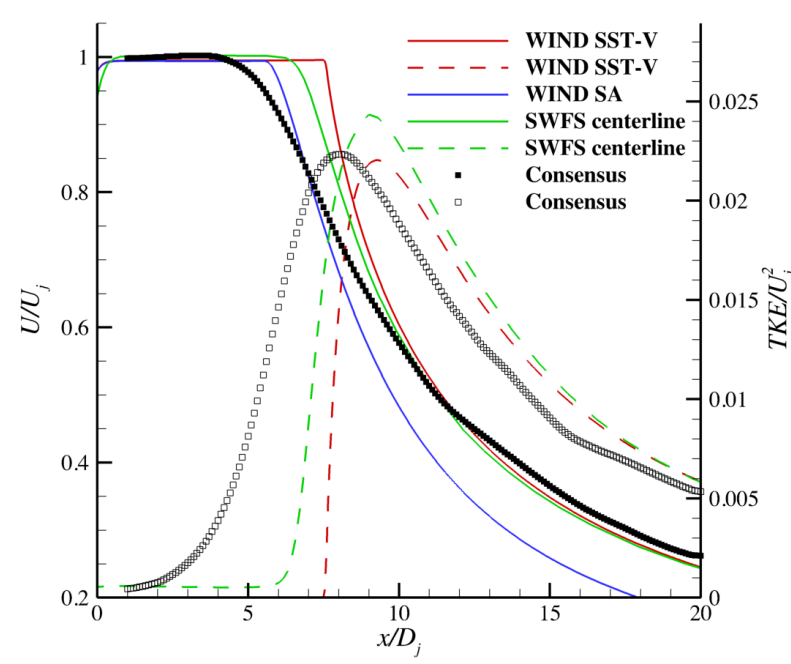

(a) Centerline

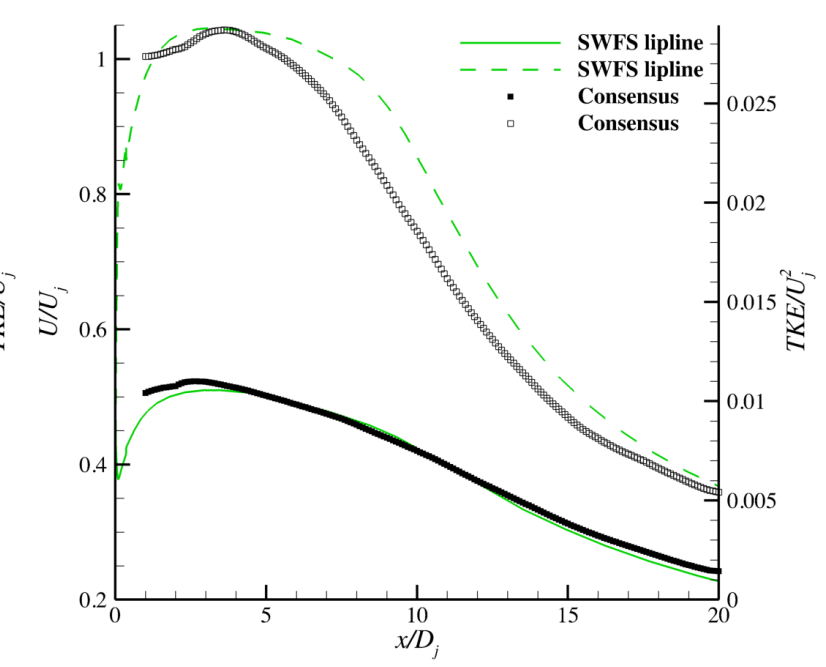

(b) Lipline

Figure 7. Comparison of solutions from WIND and SWFS codes. Mean velocity and TKE on (a) centerline (solid line), (b) lipline (dashed line).

\section{Source distribution prediction vs phased array data}

Having established the relative accuracy of the RANS input to the $m S r c$ code, we next turn to the prediction of sound source distributions, this being the first step toward computing far-field spectra. Phased arrays measure the effective sound source distributions as seen by a far-field observer at $90^{\circ}$ to the jet axis, and for this orientation are commensurate with $\mathrm{mSrc}$ predictions of source distributions integrated line-of-sight across the jet plume. Source distributions have been measured by Podboy [23] and others [24,25] over many of the flow conditions in the Tanna matrix for the SMC000 nozzle. Among other analysis, the source maps were analyzed to find the location of peak source strength for each frequency. It was discovered that when the location data were normalized by potential core length for each frequency, expressed in Strouhal number, the relationship was the same for every flow condition. The same analysis has been done for the source distributions predicted by $\mathrm{mSrc}$. shown in Figure 8 . Note that the potential core length $X_{\text {core }}$ in the SWFS flow prediction was found to be roughly $20 \%$ longer than in the data (see Figure 7), and this value was used in normalizing the axial locations of the predictions.

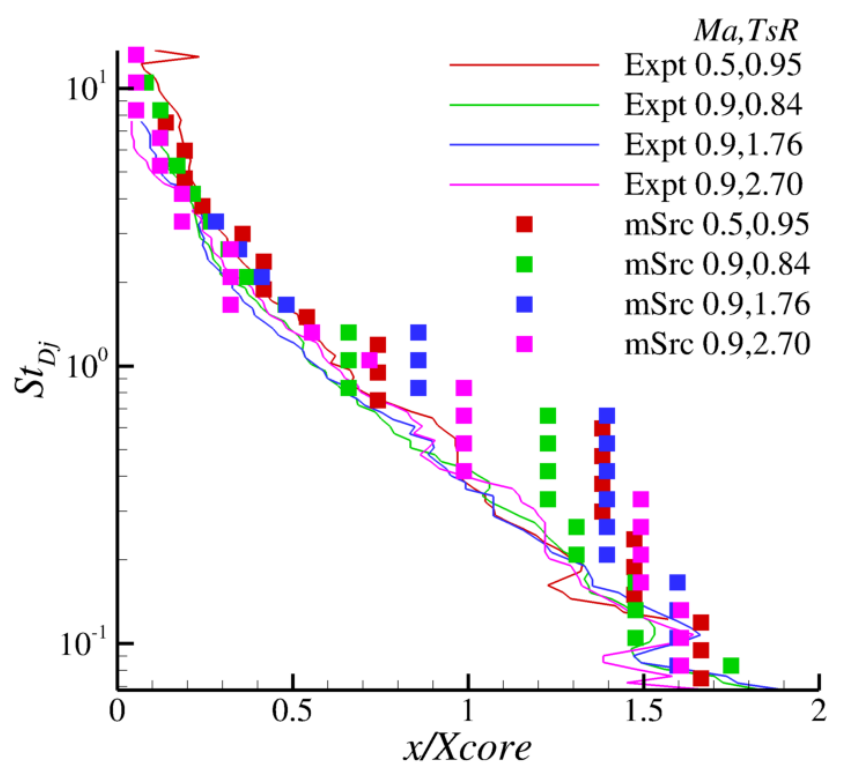

Figure 8. Peak source location vs frequency for jet noise in single-stream axisymmetric subsonic flows from the Tanna matrix, (flow conditions given in legend). Measurements of peak source locations from Ref [23]. Potential core length Xcore computed from PIV data and from SWFS separately. 


\section{Far-field predictions vs SHJAR data}

Over the course of many test campaigns on the NASA SHJAR, far-field noise has been recorded for points on the Tanna matrix using the SMC000 nozzle. In many cases, these serve as baseline cases and as a check that the facility is operating properly. The subsonic data used here is documented in [6], although it is shown here as power spectral density on $12^{\text {th }}$ octave bands instead of $1 / 3$ octave power spectra. Additional high-speed flow points were added to the matrix, using several ideally-expanded convergent-divergent nozzles, designed using method-of-characteristics to create a (nearly) shock-free jet plume. These nozzles were run [19,26] at matching combinations of $M a$ and $T s R$ to fill in the low-temperature, high-speed portions of the Tanna matrix.

The comparisons of far-field spectral directivity between $m S r c$ and experiment are extensive, and will be done by carpet plots of the spectral directivity at 100 jet diameters from the jet exit. The surface of the carpet plots is given by the predicted power spectral density, and is colored by the difference, in $\mathrm{dB}$, between the experimental data and the $m S r c$ predictions. Figure 9 is an example of this presentation. As the difference is always computed as ' $m S r c$ - data', red indicates an overprediction, and blue an underprediction. The color scales are kept at $\pm 5 \mathrm{~dB}$ for all plots in this report, with color levels being such that a green color indicates a difference within $\pm 1 \mathrm{~dB}$.

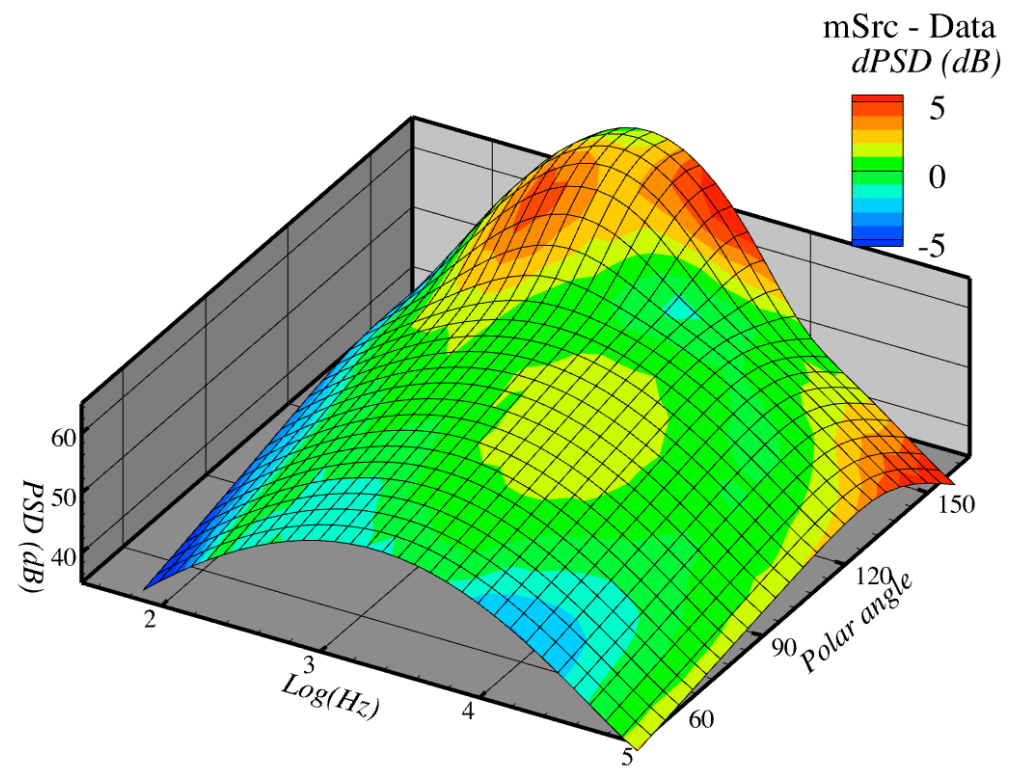

Figure 9. Example plot of spectral directivity of far-field noise, PSD (dB) vs $\log _{10}(\mathrm{~Hz})$, polar angle $\left({ }^{\circ}\right)$. Color bar is difference between $m S r c$ and data, scale $\pm 5 \mathrm{~dB}$, red is overprediction.

It is important to keep this in mind as we next present in Figure 10 a matrix of these plots, corresponding to the Tanna matrix, to show the trends in accuracy of the $m S r c$ code. In the individual plots the span of the z-axis remains the same even as it shifts to accommodate the change in overall noise. The range of flow conditions is very large, covering a range of cases from $M a=0.5$ unheated, to $M a=1.8$ with temperature ratio $T_{s} R=2.7$. Across this range of conditions peak jet noise varies from $50 \mathrm{~dB}$ to $100 \mathrm{~dB}$, and yet the error remains within $\pm 5 \mathrm{~dB}$ and much less than that for most of the range. The most significant errors are found at high acoustic Mach numbers with low temperatures (upper right), where the peak noise is overpredicted, and at low acoustic Mach numbers and high temperatures (lower left) where the peak is underpredicted. At all supersonic Mach numbers the predicted frequency of the aft peak is consistently low causing overprediction at the low frequency side of the aft peak. At acoustic Mach number 1.8 the peak amplitude is overpredicted by $4-5 \mathrm{~dB}$. For low Mach numbers there is a consistent underprediction of the noise just upstream of the aft peak where the large-scale noise begins to dominate the small-scale noise. 


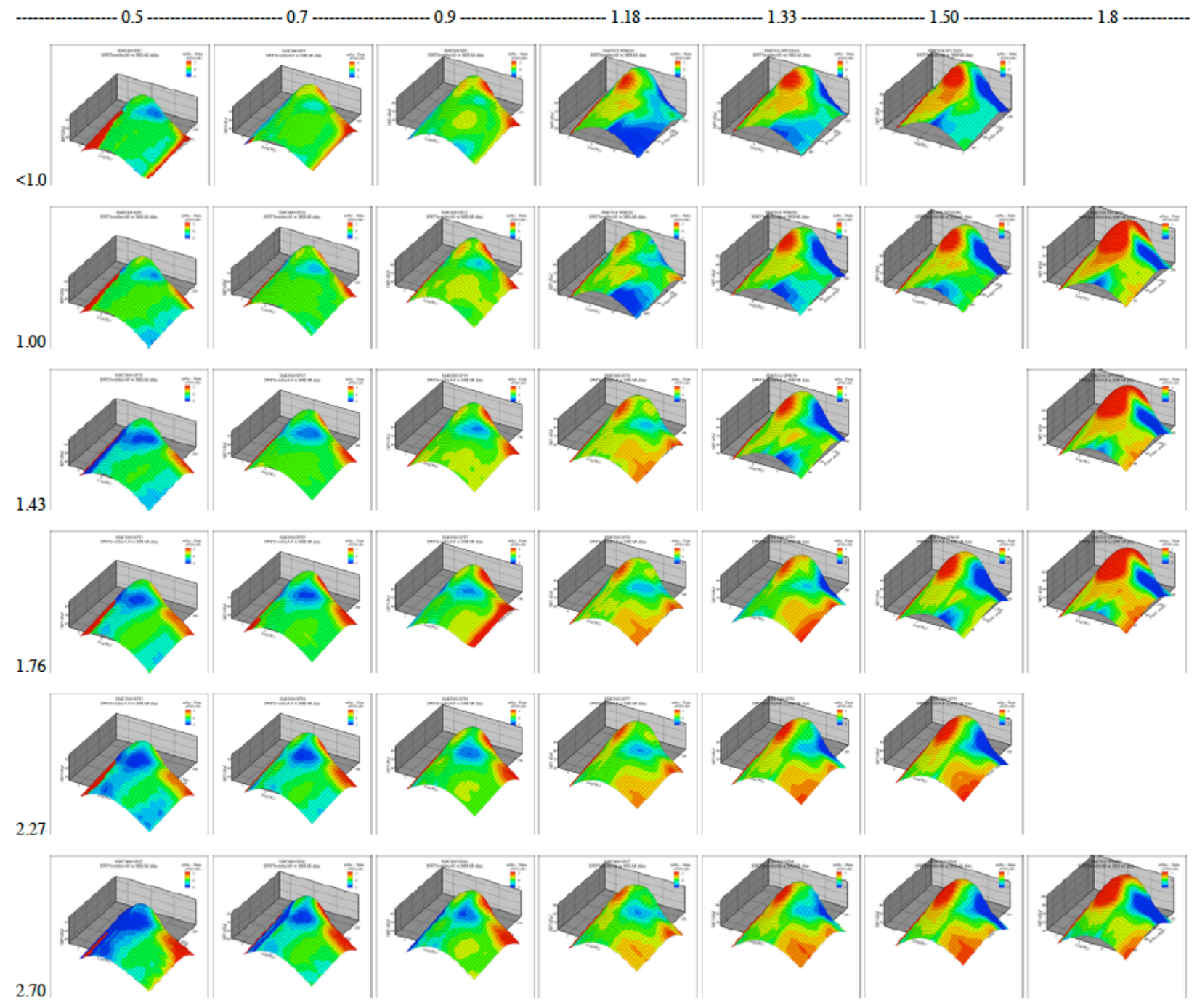

Figure 10. Matrix of spectral directivities and their errors over the expanded Tanna matrix of jet conditions, defined by acoustic Mach number (columns) $0.5<M a<1.8$, and static temperature ratio (rows) unheated $<$ $T s R<2.7$. See Figure 9 for scales of the plots.

\section{B. Dual-stream coaxial jets}

We now introduce two new aspects to the nozzle, an external centerbody and coannular streams. A few dualstream jets have been selected from tests conducted on the Nozzle Acoustic Test Rig (NATR) at NASA Glenn's AeroAcoustic Propulsion Lab, jets that strongly varied the velocity ratios of the inner and outer shear layers. Predictions of the flow, sound sources, and acoustic far-fields were made to compare with data acquired and reported in a previous test [27].

In brief, the nozzle, identified as ' $\mathrm{Cl}$ ' in reporting, is a two-stream, axisymmetric, separate flow nozzle with external plug. The tip radius of the plug is $3 \mathrm{~mm}$. The area ratio of the bypass to core streams is 2.5 . The flow matrix used followed the test design of Henderson [28], keeping the core temperature ratio (total temperature to ambient temperature) at 3.0 , the bypass temperature ratio at 1.25 , and systematically varying the pressure ratios of core and bypass. This has the effect of radically changing the velocity gradients of the two shear layers, as documented in Table 1. This variation should be a good exercise for the prediction code, strongly varying the source distributions as well as having a wide range of peak velocities. For this comparison, acoustic data was acquired with no flight stream around the plume. 
Table 1 Definition of flow conditions for Dual-stream cases

\begin{tabular}{|c|c|c|}
\hline \hline NPRc & NPRb & $\left(U_{2}-U_{1}\right) /\left(U_{2}-U_{*}\right)$ \\
\hline \hline 1.30 & 1.58 & 0.19 \\
\hline 1.50 & 1.80 & 0.31 \\
\hline 1.80 & 1.80 & 0.55 \\
\hline 2.30 & 2.30 & 0.56 \\
\hline 2.10 & 2.00 & 0.60 \\
\hline 1.50 & 1.45 & 0.62 \\
\hline 2.30 & 1.88 & 0.76 \\
\hline 1.30 & 1.22 & 0.77 \\
\hline 1.80 & 1.50 & 0.85 \\
\hline 2.10 & 1.60 & 0.92 \\
\hline 1.80 & 1.25 & 1.46 \\
\hline
\end{tabular}

\section{CFD predictions}

The impact of coannular streams is clear in the comparison of TKE distributions (Figure 11) from the dual-stream nozzle with both streams the same (single-stream mode), and with a hot core flow and cold outer flow. Close comparison of the simple single-stream plume from the SMC000 nozzle with the single-stream plume from the external plug nozzle running with both streams unheated at $\mathrm{Ma}=0.9$ showed that the external plug produced little change in the plume structure downstream of the plug. However, when there are two shear layers, the TKE at the end of the core stream potential core is significantly increased over the TKE in each shear layer. This change should make the noise source distribution very different as well.

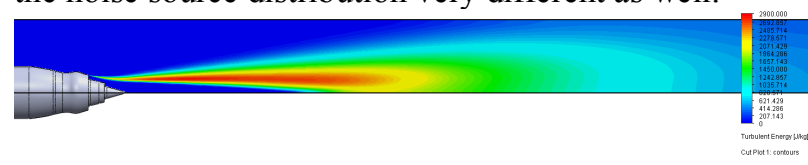

(a)

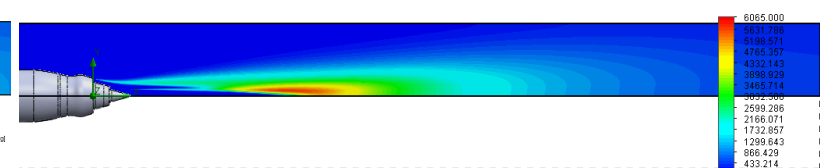

(b)

Figure 11. TKE distributions for dual stream nozzle with (a) both streams same $(M a=0.9$, unheated, peak $T K E=2900 \mathrm{~J} / \mathrm{kg})$, and (b) hot core $\left(N P R_{c}=1.8, M a=1.5, T_{s} R=2.56\right)$ and $\operatorname{cool}$ bypass $\left(N P R_{b}=1.5, M a=0.8, T_{s} R=1.1\right)$ (peak $T K E=6000 \mathrm{~J} / \mathrm{kg}$ ).

\section{Source distribution predictions vs phased array data}

The phased array data used in the following section was acquired at $90^{\circ}$ to the jet axis, plug tip, at a distance of 2 meters. In Figure 12, pairs of source distributions are shown for six frequencies. At each frequency, phased array measurements are shown superimposed on a photo of the nozzle from the array's perspective, stacked atop $m S r c$ predictions below. The dynamic range of the phased array data is very small $(1 \mathrm{~dB})$ compared with the $10 \mathrm{~dB}$ range of the predictions. The progression of the source distribution from downstream to upstream, and particularly the abrupt shift from the peak TKE region downstream to the nozzle, is similar in the two methods, if not exactly the same. 


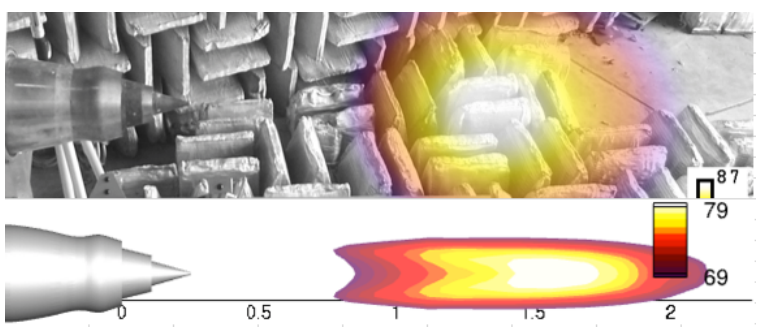

$50 \mathrm{~Hz}$
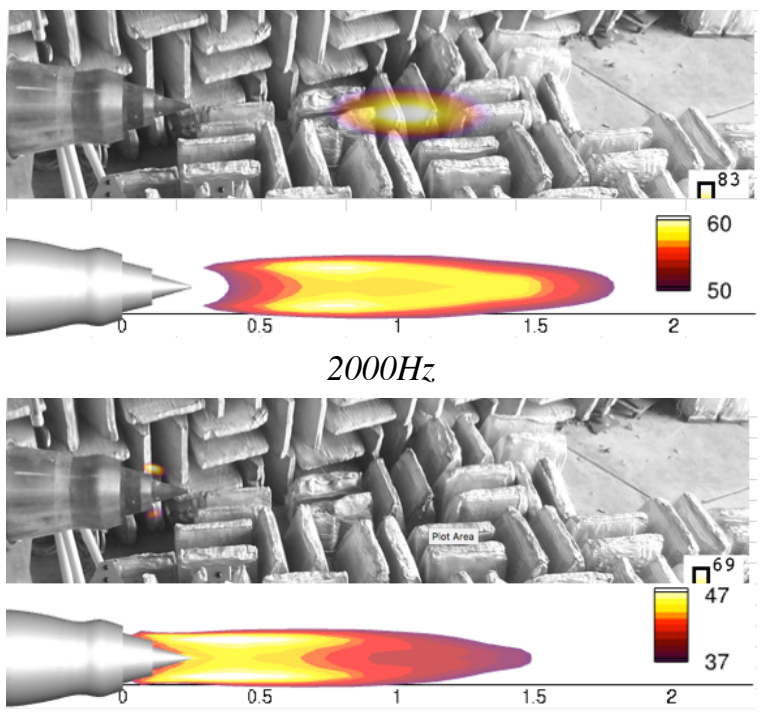

$8000 \mathrm{~Hz}$

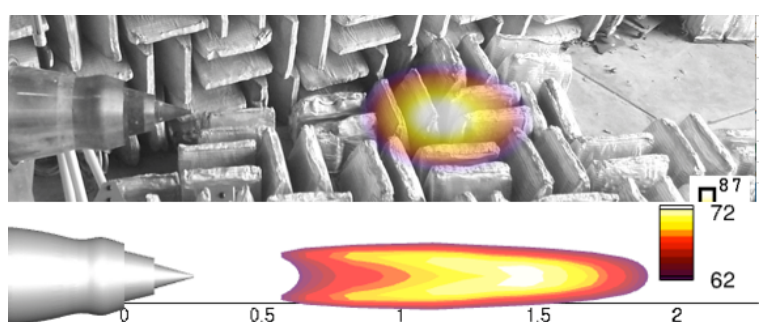

$1000 \mathrm{~Hz}$

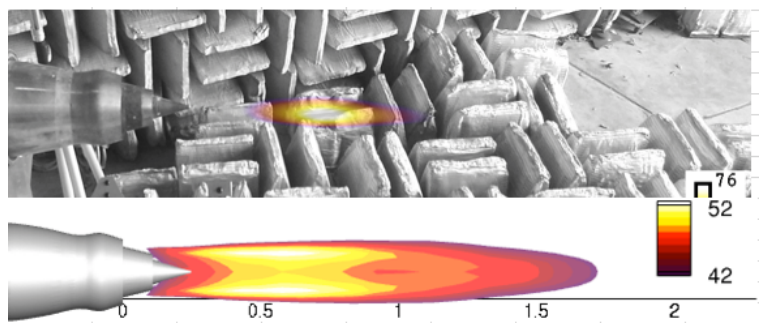

$4000 \mathrm{~Hz}$

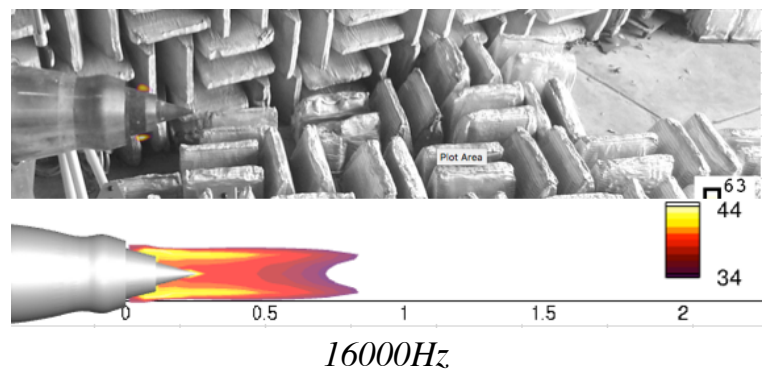

Figure 12. Maps of sound sources in the jet plume at different frequencies as measured by a phased array (top) and as predicted by $m S r c$ (bottom). Nozzle $\mathrm{C} 1, N P R_{c}=1.3, N P R_{b}=1.58$.

\section{Far-field predictions vs NATR data}

The comparison of far-field predictions with data are again made using a matrix of carpet plots. These have the same axes and colorscales as Figure 9 and Figure 10, and are arranged in a matrix by core nozzle pressure ratio (NPRc) in columns and bypass nozzle pressure ratio $(N P R b)$ in rows.

Generally, $m S r c$ consistently missed the peak angle of the aft peak in the spectral directivity for the dual stream jets by about $5^{\circ}$, as evidenced by the red coloration on the last two angles. This can probably be attributed to the inadequacy of the method used to estimate equivalent temperature ratio of the jet, a factor used in modeling the directivity of the peak (Equation 7).

$m S r c$ also overpredicted the highest frequencies in several cases, although the trend is a little hard to fathom. Here's where the ability of $m S r c$ to backtrack through source distributions is useful to determine the cause of spectral characteristics. The three-dimensional source density as predicted by $m S r c$ for the flow with $N P R c=1.3, N P R b=1.22$ (top left in Figure 12), was examined, and a strong contribution from TKE on the downstream portion of the plug was noted. Figure 14a shows a cross-section of the source density where the high amplitude source density can be noted. Figure $14 \mathrm{~b}$ then shows the source density cross-section if $m S r c$ is told that the plug is $1 \mathrm{~mm}$ larger in radius than the CFD solution, omitting much of the TKE of the predicted separation region on the plug. The source density remaining is much lower, and the predicted high frequency far-field noise is greatly reduced. This exercise suggests three things: separated flow on external plugs can be a very loud high frequency source, the experiment probably did not have as much separation as predicted by the CFD, and $\mathrm{mSrc}$ is very handy for understanding how nozzle geometry influences noise. 


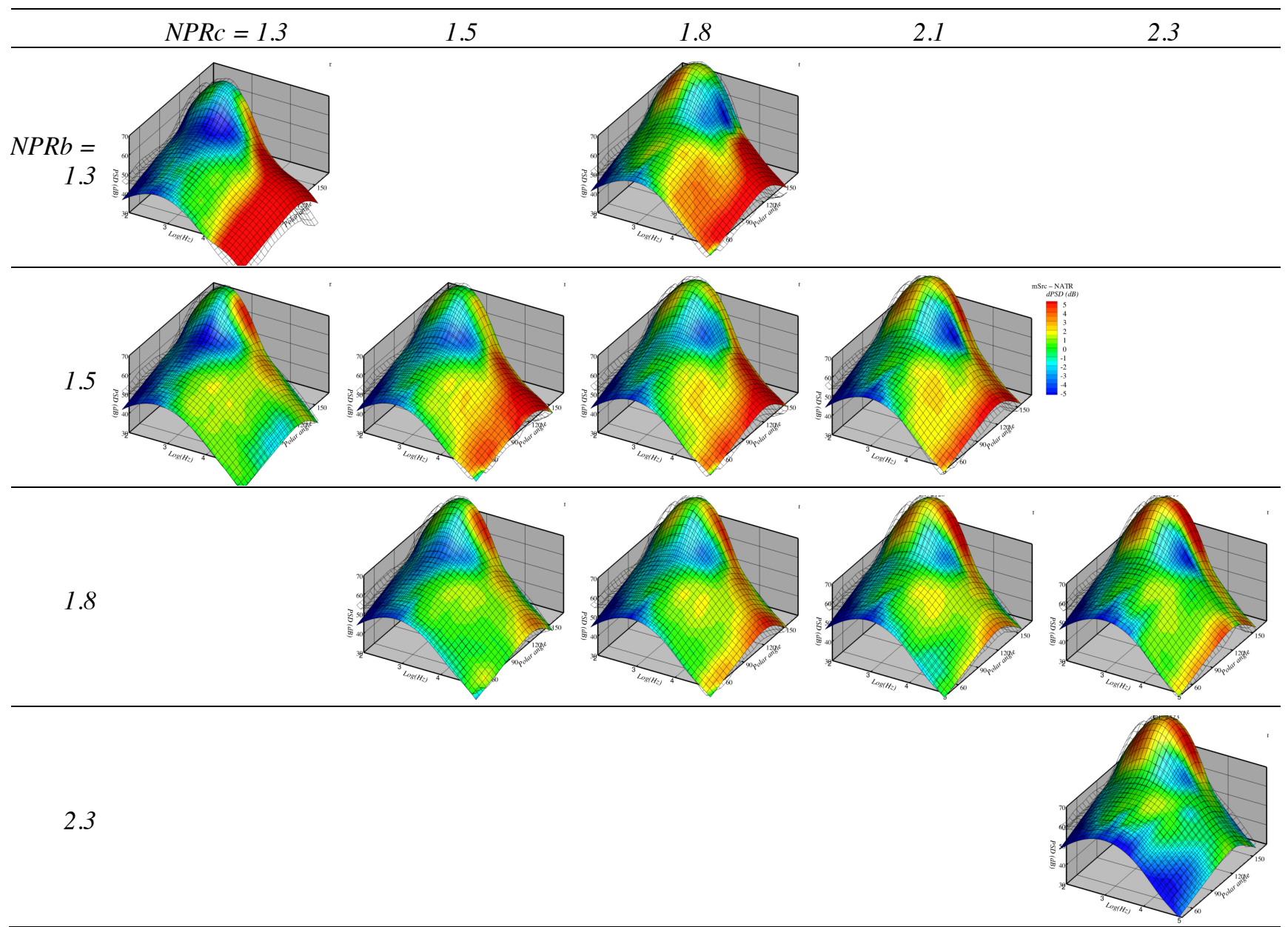

Figure 13. Spectral directivity of far-field noise from coaxial jets. Core total temperature ratio $=3$, bypass total temperature ratio $=\mathbf{1 . 2 5}$. Nozzle pressure ratios for core and bypass given by labels at edges of matrix Color bar is difference between $m S r c$ and data, scale $\pm 5 \mathrm{~dB}$. 

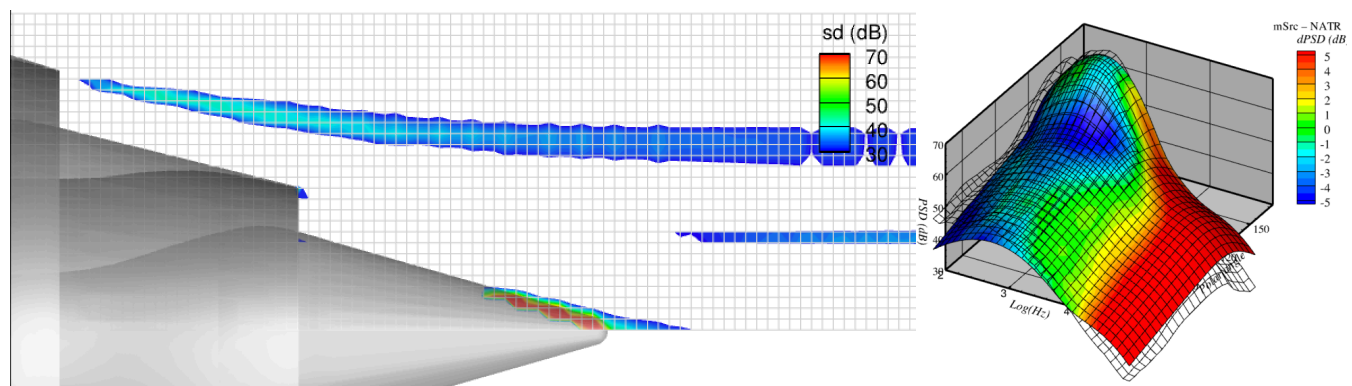

(a) original full integration region

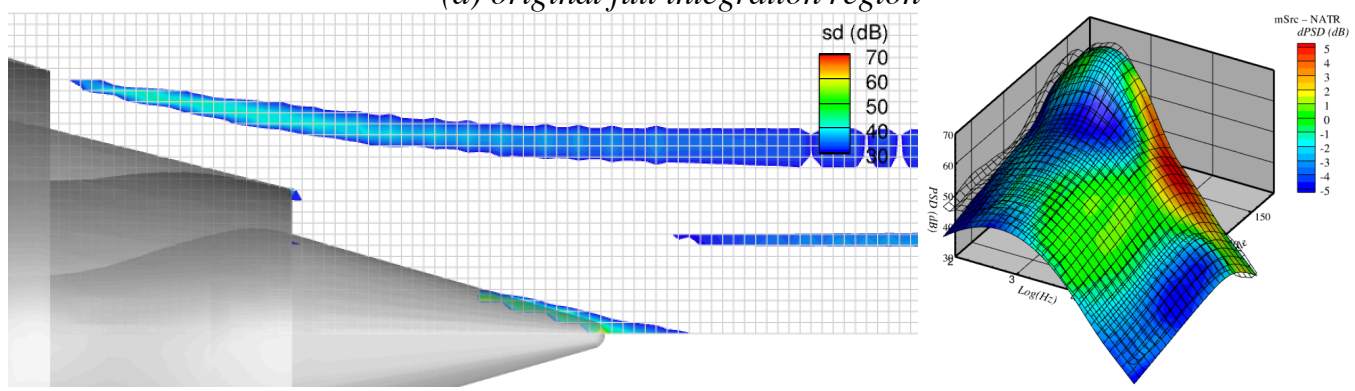

(b) modified integration region omitting Imm annulus around plug.

Figure 14. Source density cross-section and far-field spectral directivity for nozzle C1, $N P R c=1.3, N P R b=1.22$. Note difference in source density on plug and impact on high frequency far-field noise.

\section{Nozzles with enhanced mixing features}

Returning to single-stream jets, a set of simple chevron nozzles were created in 2003, and were used in tests $[24,29,30]$ over subsequent years. Two of these nozzles, SMC001 and SMC006, have been used for validating other prediction methods, and will be used here to test the ability of $m S r c$ to capture the acoustic effects of enhanced mixing devices. Both have six chevrons, SMC001 having a chevron penetration angle of $5^{\circ}$ to the jet axis, and SMC006 a penetration angle of $18^{\circ}$.

\section{CFD predictions vs PIV data}

As mentioned before, RANS codes rarely exactly match jet flows as measured by PIV. SWFS and FUN3D are no exception, but the errors are systematic, and have been accounted for in $m S r c$. To better compare the SWFS results with PIV data in Figure 15, the SWFS values for TKE were divided in half. The elongation of fingers in the jet crosssection by axial vortices of the chevrons is well predicted, and the TKE values, once adjusted, match over most of the jet within $20 \%$. The predicted flow for the chevron nozzle actually matches the data better than predictions for the round nozzle. 

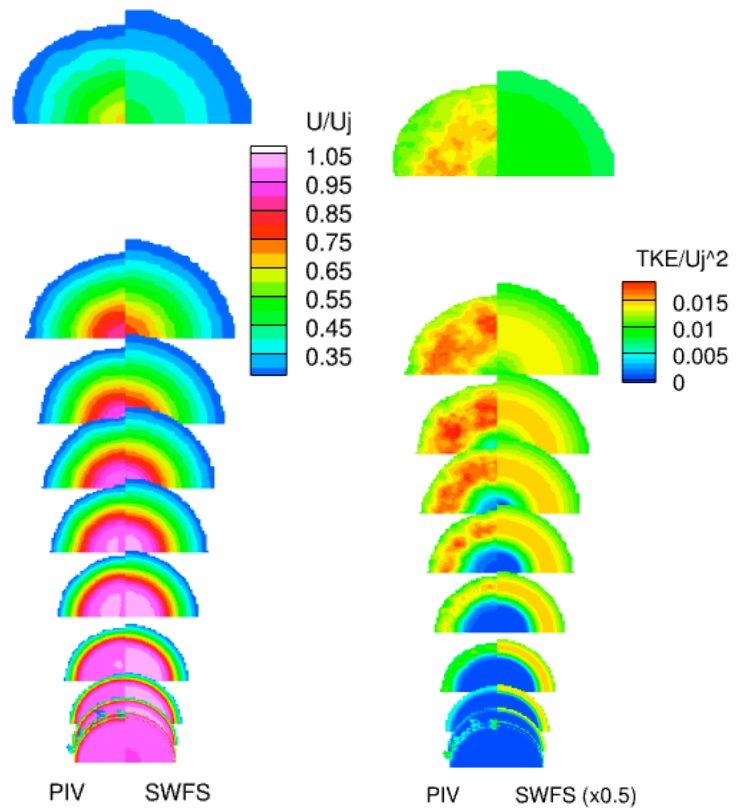

SMCOOO
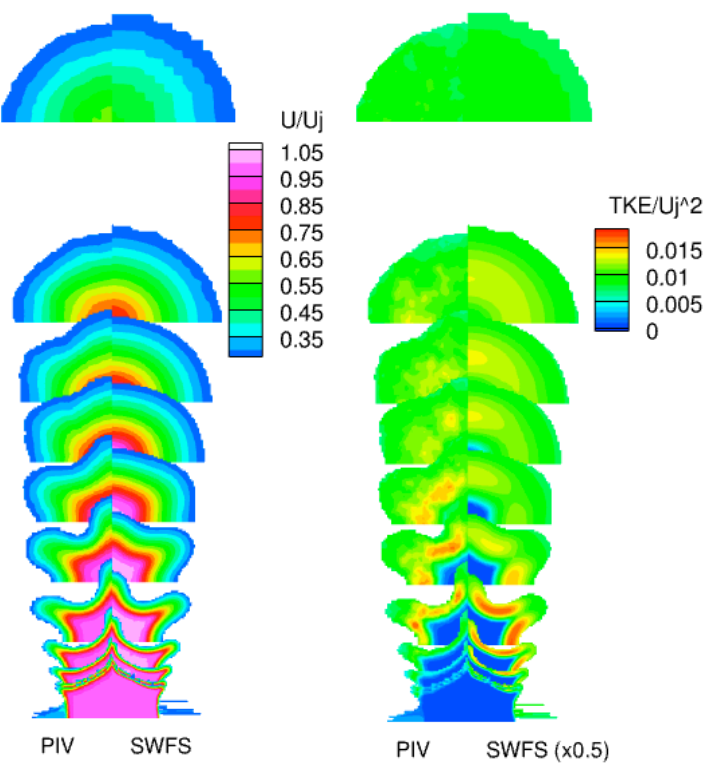

SMCOO1

Figure 15. Comparisons of mean axial velocity and TKE, acquired by PIV and predicted by SWFS, for the round SMC000 and chevron SMC001 nozzles operating unheated with Ma = 0.9 (Tanna setpoint 7).

\section{Source distribution predictions vs phased array data}

In 2009, a phased array measured source distributions of a number of nozzles on the NASA SHJAR, including the SMC000 and SMC001 nozzles. Portions of Figures 15 and 17 from the NASA Technical Memo [31] which show the $25 \mathrm{kHz}-50 \mathrm{kHz}$ source maps for these two nozzles at Tanna setpoint 7, are reproduced in Figure 16. Corresponding source maps, created by integrating the source distributions across the jet, are shown below them, comparing the distributions. Both measurements and predictions are for an observer at right angles to the jet. What should catch the eye is how the source distribution of the chevron nozzle, when integrated along the z-axis (aligned with a valley of the 6-chevron nozzle) has three streaks aligned with the valleys of the chevrons in both the measured and predicted distributions. Distributions from round jets tend to align with the shear layer and hence have only two streaks. When the SMC001 source distribution is integrated along the y-axis (aligned with the chevron tips), the predicted six streaks of sources align to produce two broad streaks, an effect unfortunately not captured during the experiment as only one nozzle orientation was measured. However, note the scales on the color bars of the plots. In the measurements, the peak source density is $6 \mathrm{~dB}$ higher for the SMC001 plot than the SMC000 plot. In the $m S r c$ predictions, the peak source density is only $3 \mathrm{~dB}$ higher.

Figure 17 presents the same comparisons, only this time at $2 \mathrm{kHz}$, near the peak frequency of the jet at broadside angles. Here, the effect of chevrons on the source distribution, according to the phased array, is to move the sources closer to the nozzle and make them slightly weaker. According to the $m S r c$ predictions, the chevrons did not move the sources axially, but did reduce their strength considerably. 


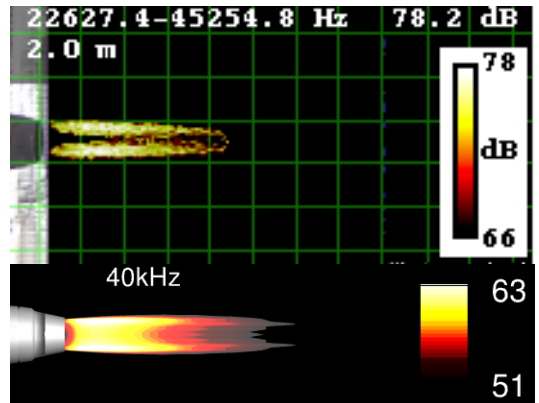

SMCOOO
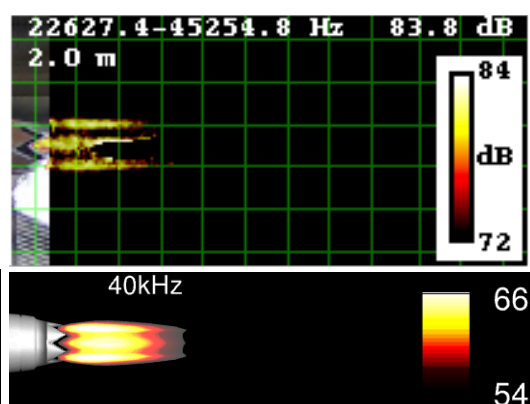

SMC001 (xy-plane)

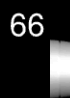

54
$40 \mathrm{kHz}$

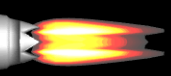

Figure 16. Source distributions at $50 \mathrm{kHz}$ of (a) round nozzle SMC000 and (b) chevron nozzle SMC001, xyplane, and (c) SMC001, xz-plane. Upper row: as measured by phased array ( $(r e f)$ ). Lower row: as predicted by $\mathrm{mSrc}$. Jet operating unheated with $\mathrm{Ma}=0.9$ (Tanna setpoint 7 ).

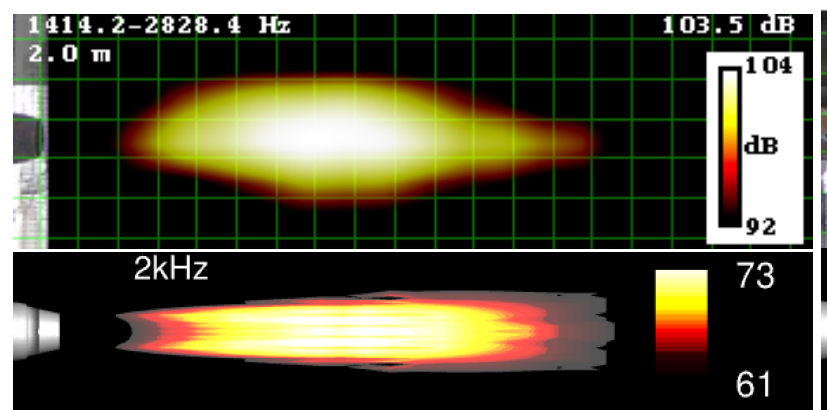

SMCOOO

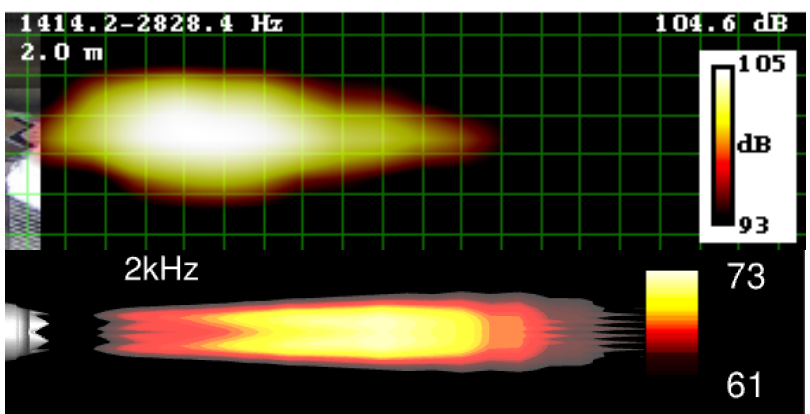

SMC001 (xy-plane)

Figure 17. Same as Figure 16, but for $2 \mathrm{kHz}$ source distributions.

\section{Far-field predictions vs SHJAR data}

The performance of $\mathrm{mSrc}$ at predicting the far-field noise impact of chevrons is documented in Figure 18. The spectral directivities predicted by $\mathrm{mSrc}$ for the round SMC000 nozzle, the $5^{\circ}$ penetration SMC001 nozzle, and the $18^{\circ}$ penetration SMC006 nozzle are shown, colored by the difference between prediction and data. While $\mathrm{mSrc}$ does predict an increase at high frequencies when the chevrons are in place, it underpredicts the increase. $m S r c$ does get the proper reduction in the aft peak.

To understand the source of the discrepancy, start by looking closely at phased array results. In both experiments and prediction, the high frequency sources shift upstream, but as noted above, the predicted source densities for the chevron nozzle is only $3 \mathrm{~dB}$ stronger than the round nozzle. The experiment finds it $6 \mathrm{~B}$ stronger. Two possible explanations are put forth: (i) the chevron-induced increase in TKE near the nozzle isn't captured by the CFD, or (ii) the TKE becomes more efficient at producing noise.

Pursuing the first possibility, careful examination of the CFD in Figure 15 shows that, although the CFD doesn't exactly capture the PIV results, they don't miss by $20 \%$ or more needed to explain the shortfall. Also, these cases were done both with SWFS and with a much finer-resolved FUN3D input, and the results of the using the two CFD results were within $1 \mathrm{~dB}$ of each other.

As to the second possibility, earlier work [32,33] suggested that chevrons change the TKE anisotropy, and that this is responsible for the enhanced noise beyond just TKE levels. Indeed, when the ratio of axial to transverse components of the turbulent velocity are investigated in PIV data, the transverse component comprises a much larger fraction of the total TKE in the first diameter of the chevron nozzles. If turbulent anisotropy is key to properly predicting chevron noise, then a more elaborate CFD code using Reynolds Stress turbulence modeling will have to be used to predict this anisotropy for the source model. 


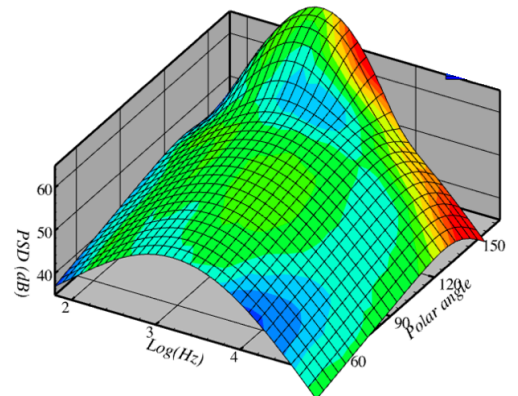

SMCOOO

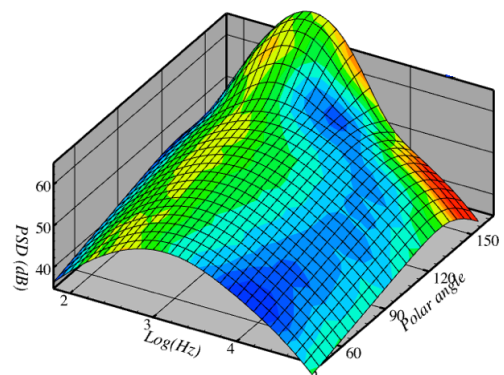

SMCO01

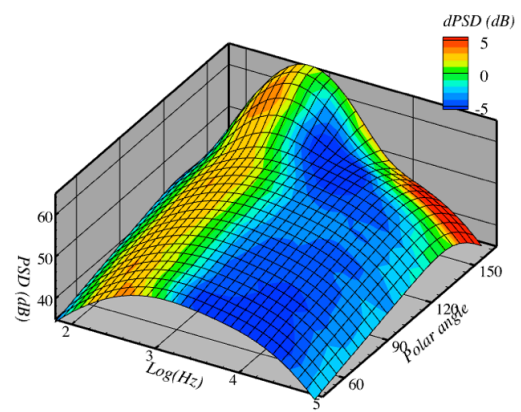

SMC006

Figure 18. Spectral directivity of far-field noise from single-stream jets from chevron nozzles, unheated, Ma=0.9 flow. Color bar is difference between $\mathbf{m S r c}$ and data, scale $\pm 5 \mathrm{~dB}$.

\section{Jets in proximity to surfaces}

To obtain a first-order answer on the effect of surfaces near the jet, a simple Fresnel integral method is used by $m S r c$ to compute the shielding or reflection of the jet noise sources, making the large assumption that the point-source paradigm holds for jet sources. The effectiveness of this model was evaluated by comparing predictions with data acquired in a series of jet-surface interaction tests [34]. Comparisons of CFD will be made with PIV data from Brown \& Wernet [35]. Comparisons of sound will be made with data described in Brown [36], and in particular with the noise of the jet after the spatially coherent trailing edge dipole sound has been removed.

In the jet-surface interaction tests where the surface represented a semi-infinite plate, two surface parameters were varied relative to the baseline SMC000 nozzle: streamwise distance $x_{E}$ between nozzle and surface trailing edge, and standoff distance $h_{E}$ between the nozzle lip and the surface. A representative subset of these parameters was chosen for this validation exercise: $x_{E} / D=\{0,0.5,2.5\}$ and $h_{E} / D=\{1.4,2,4,6\}$. All cases were run with an unheated, $M a=$ 0.9 (Tanna setpoint 7) flow.

\section{CFD predictions vs PIV data}

RANS solutions were obtained for all the surface configurations using SWFS, even though the surface had little impact on the plume at $h_{E} / D>1$. This was confirmed by PIV data. Significant impacts were measured and predicted for smaller standoffs, especially as the plate extended far downstream. Figure 19 presents comparisons of the TKE fields, measured and predicted, for a $x_{E} / D=6$ surface with no standoff and with $0.5 D$ standoff. The biggest shortcoming of the SWFS solution relative to PIV measurements is in the $h_{E} / D=0$ configuration. SWFS does not predict the amplification of the shear layer TKE downstream of the trailing edge, where the TKE reaches levels significantly higher than is measured in a conventional shear layer. TKE $/ U_{\mathrm{j}}^{2}$ usually peaks around 0.023 ; the peak $\mathrm{TKE} / U_{\mathrm{j}}^{2}$ a few diameters downstream of the surface is over 0.03 . It is postulated that the fresh shear layer starting at the trailing edge of the surface has its turbulent growth amplified by the TKE already present in the plume, an effect not captured by the turbulence models of the CFD that operate on only on the local shear.

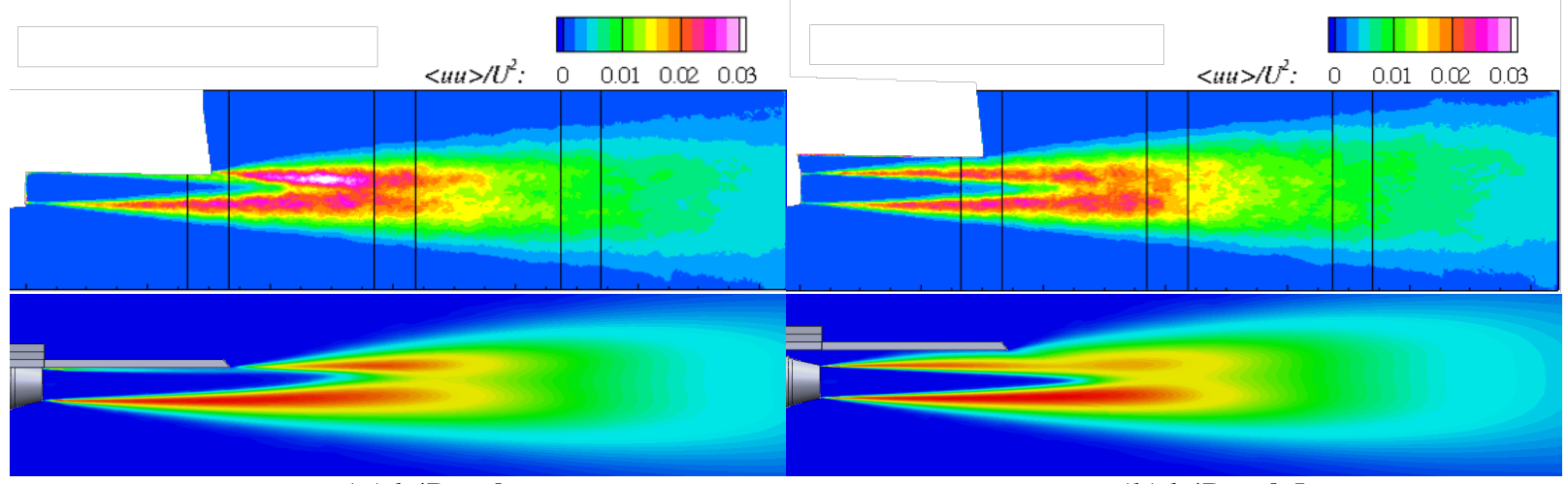

(a) $h_{t} / D=0$

(b) $h_{\S} / D=0.5$

Figure 19. Comparisons of TKE in symmetry plane for round jet near flat plate. Plate extends 6 diameters aft of jet exit, stands off from lipline (a) by 0 and (b) by 0.5D. PIV data on top row, SWFS predictions on bottom row. 


\section{Far-field predictions vs SHJAR data}

Far-field acoustic predictions are compared with data for observers on the shielded side of the surface. The results are given in terms of amount of shielding predicted versus amount of shielding measured. In both predictions and experiments the shielding is given by subtracting the noise of the isolated baseline jet from the noise measured with the surface in place. For the experimental data, this was done after the spatially coherent, low frequency noise from the flow interacting with the trailing edge was removed using the extraction technique described in Brown [36]. The matrix of points in Figure 20 covers the range of surface dimensions from the nozzle lip being flush with a very short surface to the nozzle being unreasonably far off an unreasonably long surface. The amount of shielding measured was up to $10 \mathrm{~dB}$ for the longest surface. As seen in the matrix of cases, the shielding model in $m S r c$ generally overpredicted the shielding. It is worth looking in detail at the shielding for a few cases.

When the jet was on the surface $\left(h_{E} / D=0\right), m S r c$ predicted a small shielding when in fact the measurements usually showed a slight increase. The prediction compared more favorably as the surface was moved away from the plume, as seen in Figure 21. Even so, at the highest frequencies the shielding model in $\mathrm{mSrc}$ predicted a very strong increase in shielding with frequency, while the data generally didn't fall of as quickly. It seems likely that the problem with the prediction for surfaces very near the nozzle lies with the inability of the CFD to predict the increase in TKE of the new shear layer generated at the trailing edge of the surface, as noted above. This is a different noise source than the trailing edge dipole, has nothing to do with the shielding model, and should be predicted by $m S r c$ if the CFD were accurate.

The only case where $m S r c$ underpredicted the shielding was for longer surfaces with the largest standoff. As seen in Figure 22, when the surface is $2.5 \mathrm{D}$ outside the flow the measurements show shielding starting at a lower frequency than predicted by $m S r c$, especially for upstream angles.

The deficiency of $\mathrm{mSrc}$ in predicting the shielding produced by surfaces near the jet is not too surprising. A number of assumptions have been made, starting with the assumption that the acoustic sources can be treated as independent point sources. There is no accounting for flow convection, a feature of a proper Green's function for this problem. Hydrodynamic forces on the surface becoming sources are not accounted for. When comparing the flow predictions to PIV there are substantial shortcomings in the RANS solution even if the other assumptions were valid. This is clearly an area ripe for new ideas, as for now an exhaust system designer has to discount much of the shielding benefit predicted while doing their work.

$h_{\text {f }} / D=0$
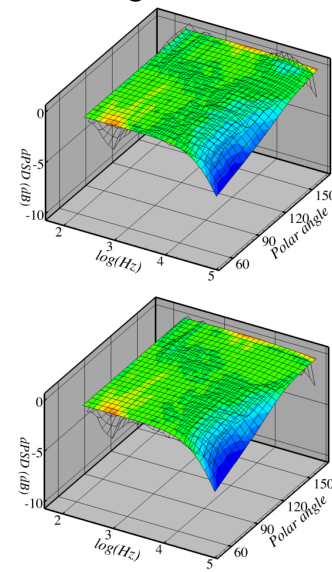

$h_{t} / D=0.5$

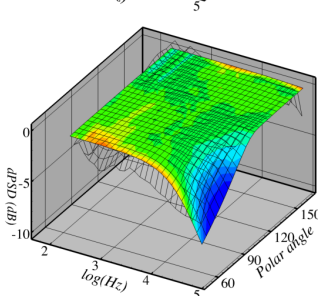

$x_{\varepsilon} / D=0.7$
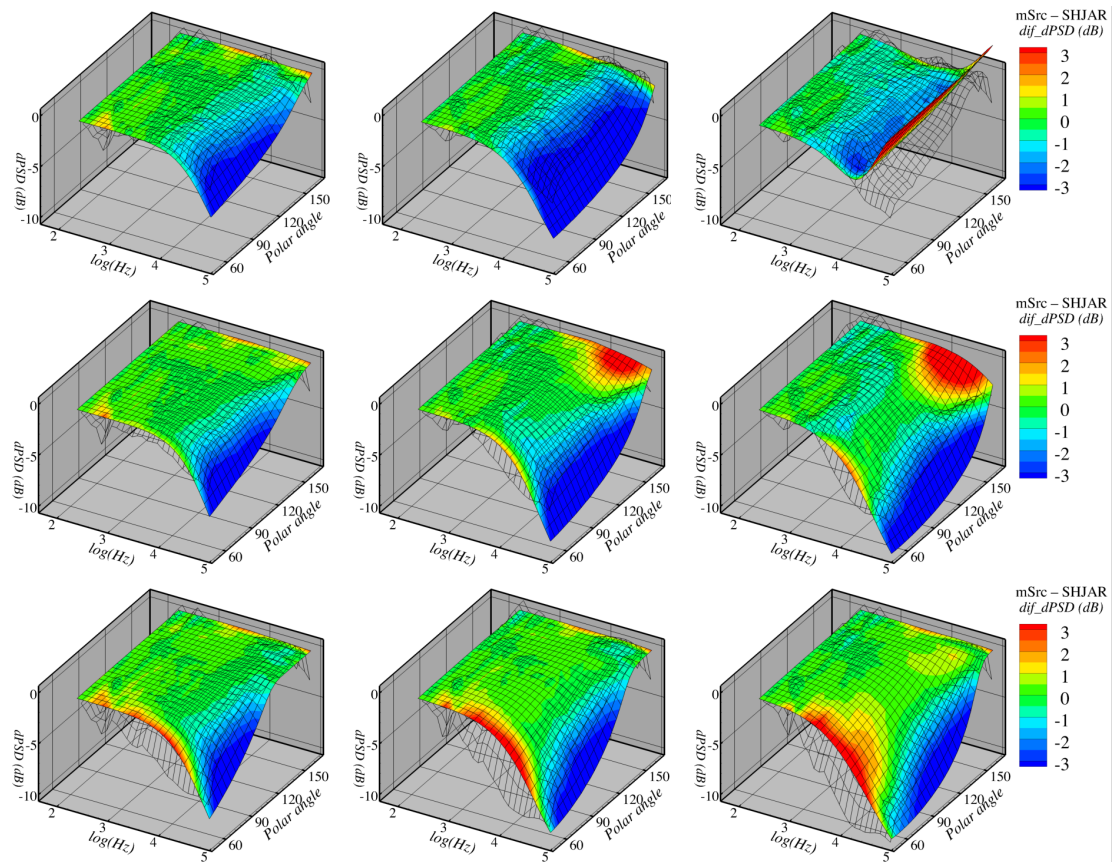

$x_{\diamond} / D=4$

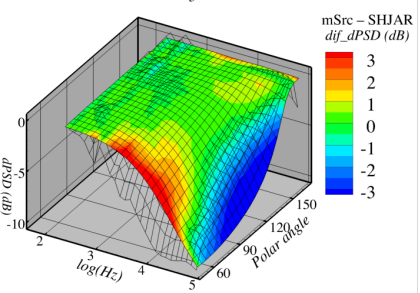

$x_{\mathrm{A}} / D=6$

Figure 20. Spectral directivity of shielding of far-field sound from single-stream unheated, $M a=0.9$ jet near flat plate. Surface shape is difference between jet with and without plate. Color bar is difference between mSrc and data, scale $\pm 5 \mathrm{~dB}$. Observer is on shielding side of plate. Plate length downstream of nozzle exit is $x E$, distance between nozzle lip and plate surface is $h E$. 

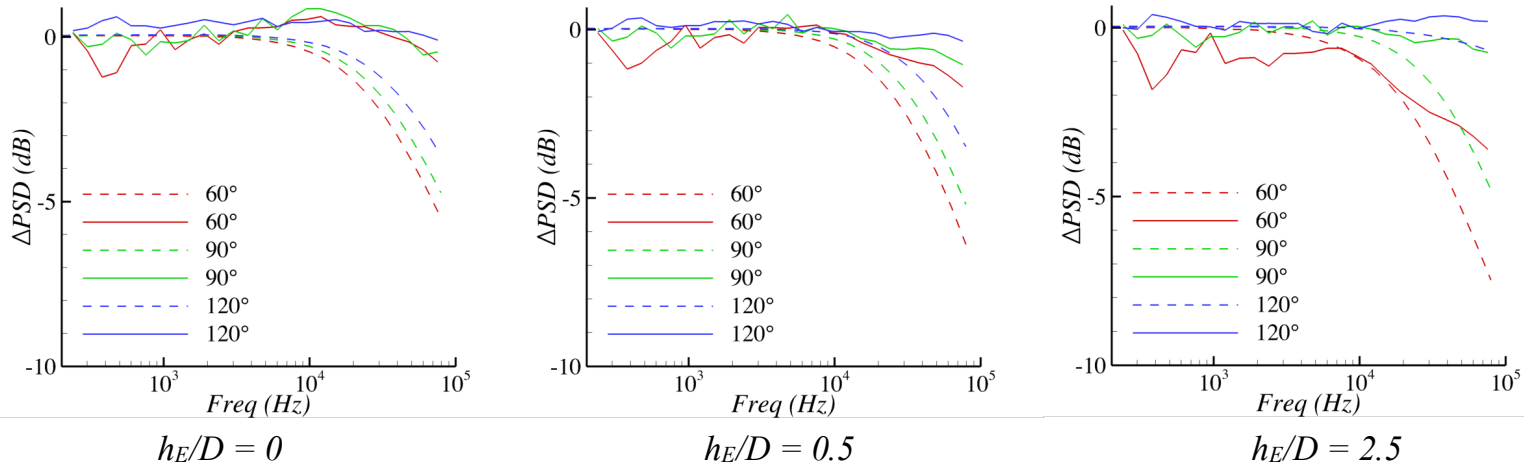

Figure 21. Shielding predicted (dashed line) vs shielding (solid line) measured for surface with length $x E / D=2$.
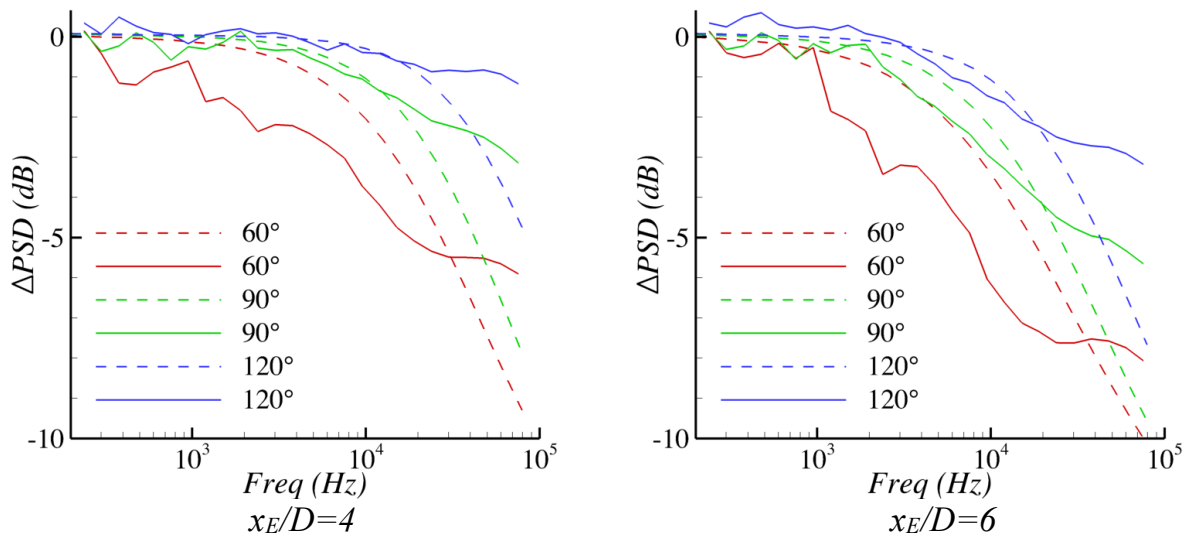

Figure 22. Shielding predicted (dashed line) vs shielding (solid line) measured for surface positions outside of flow $h_{E} / D=2.5$.

\section{Summary}

A new method has been developed for prediction of jet exhaust noise using input from Reynolds-Averaged NavierStokes predictions of the turbulent flow. The method is loosely based on acoustic analogy theory, using a simplified source model and substituting models for the directivity of the sources for the usual Green's function to prescribe the propagation of the sources to the far-field observers. The main advantage of this approach is reducing sensitivity to the CFD input, allowing direct use of unstructured-grid codes, including embedded boundary codes, which in turn leads to greatly reduced time for the predictions.

This paper starts with motivation for a method that marries insights from acoustic analogies to insights from myriad jet noise experiments. The method is then described, first covering the source models applied to the CFD input, and then in descriptions of the propagation models. The latter half of the paper shows applications of $m S r c$ to jet configurations and flows documented in years of NASA tests. In most cases, representative cases of flow and phased array source measurements are compared with the $\mathrm{mSrc}$ predictions before the full range of far-field noise predictions are directly compared with data. The $m S r c$ code is exercised on:

- $\quad$ single-stream round jet flows, covering acoustic Mach numbers from 0.5 to 1.8 and static temperature ratios from unheated to 2.7 ,

- a range of dual-stream jets resembling conventional subsonic aircraft engines,

- several single-stream jets with chevron nozzles of different design,

- single-stream round jet in proximity to various flat plate representations of installations.

The spectral directivity of the isolated, single-stream jets were predicted within $\pm 2 \mathrm{~dB}$ over the wide range of flows, beginning to show large deficiencies as $M a$ became greater than 1.5. The spectral directivity of the dual stream jets were also predicted within a few $\mathrm{dB}$ over a wide range of conditions. One area of discrepancy seems to be in whether the external plug had separation or not, a feature made apparent when the predicted source distribution was examined. The effect of chevrons on the round nozzle was not predicted to be as strong as measured, although the low frequency benefits were captured. This is an area of future development as it is key to many potential applications of $m S r c$. The 
shielding and reflection predicted by the crude barrier theory model was too strong of an effect. A better model might be developed, or this may have to be accounted for externally when using this quick method of noise prediction.

The validation effort shows where the $m S r c$ method can be used, and to what accuracy it should be trusted. The method does not predict broadband shock noise, screech, or surface-induced dipole noise sources. But for many design applications, limitations in accuracy of the $m S r c$ method are easily outweighed by the decreased turnaround time, allowing quick exploration of concepts. Its ability to relate turbulent flow features to source distributions and to farfield noise makes $m S r c$ a true design tool.

\section{Acknowledgements}

This work was seeded by years of discussions with colleagues such as Marvin Goldstein, Abbas Khavaran, and Stewart Leib. Much of the source modeling contained in $m S r c$ was inspired by the work of Dr. Khavaran, and Dr. Leib has been very helpful in clarifying aspects of Green's functions and how they relate to the empirical modeling contained in this work. The collaboration of many experimentalist colleagues has also been required for this work: Mark Wernet for PIV data from many test campaigns; Cliff Brown for his work on jet-surface interaction, including processing of data used in this report; Gary Podboy for his phased array data. The NASA Supersonic Technology Project provided support for the work as well as motivation as it seeks to make supersonic commercial flight a reality.

\section{References}

${ }^{1}$ Gillian, R. E., “Aircraft noise prediction program user's manual," NASA-TM-84486, 1982. https://ntrs.nasa.gov/search.jsp?R=19830007879.

${ }^{2}$ Leib, S. J., and Goldstein, M. E., "Hybrid Source Model for Predicting High-Speed Jet Noise," AIAA Journal, vol. 49, 2011, pp. 1324-1335. doi:10.2514/1.J050707.

${ }^{3}$ Khavaran, A., Bridges, J., and Georgiadis, N., "Prediction of Turbulence-Generated Noise in Unheated Jets. Part 1; JeNo Technical Manual (Version 1.0)," NASA/TM-2005-213827/PT1, 2005. https://ntrs.nasa.gov/search.jsp?R=20050207369.

${ }^{4}$ Khavaran, A., and Bridges, J., "An Empirical Temperature Variance Source Model in Heated Jets," NASA/TM-2012217743, 2012. https://ntrs.nasa.gov/search.jsp?R=20120015916.

${ }^{5}$ Leib, S. J., "Modeling Sound Propagation Through Non-Axisymmetric Jets," NASA/CR-2014-218107, 2014. https://ntrs.nasa.gov/search.jsp?R=20140012697.

${ }^{6}$ Brown, C. A., and Bridges, J., "Small Hot Jet Acoustic Rig Validation," NASA/TM-2006-214234, 2006. https://ntrs.nasa.gov/search.jsp?R=20060013451.

${ }^{7}$ Tanna, H. K., Dean, P. D., and Burrin, R. H., The Generation and Radiation of Supersonic Jet Noise. Volume 3. Turbulent Mixing Noise Data, LOCKHEED-GEORGIA CO MARIETTA, LOCKHEED-GEORGIA CO MARIETTA, 1976. http://www.dtic.mil/docs/citations/ADA032882.

${ }^{8}$ Bridges, J., and Brown, C., "Validation of the Small Hot Jet Acoustic Rig for Aeroacoustic Research," 11th AIAA/CEAS Aeroacoustics Conference, American Institute of Aeronautics and Astronautics, 2011. doi:10.2514/6.2005-2846.

${ }^{9}$ Guo, Y., Pope, D. S., Burley, C. L., and Thomas, R. H., “Aircraft System Noise Shielding Prediction with a Kirchhoff Integral Method," 23rd AIAA/CEAS Aeroacoustics Conference, American Institute of Aeronautics and Astronautics, 2017. doi:10.2514/6.2017-3196.

${ }^{10}$ Khavaran, A., "Jet Surface Interaction - Scrubbing Noise in a Transversely Sheared Mean Flow," International Journal of Aeroacoustics, vol. 14, Aug. 2015, pp. 373-412. doi:10.1260/1475-472X.14.3-4.373.

${ }^{11}$ Khavaran, A., "Green's Function Applicable to Turbofan Exhaust Noise in Jets with an External Center-Body," 2018 AIAA/CEAS Aeroacoustics Conference, American Institute of Aeronautics and Astronautics, 2018. doi:10.2514/6.2018-3613.

${ }^{12}$ Goldstein, M. E., Leib, S. J., and Afsar, M. Z., "Generalized rapid-distortion theory on transversely sheared mean flows with physically realizable upstream boundary conditions: application to trailing-edge problem," Journal of Fluid Mechanics, vol. 824, Aug. 2017, pp. 477-512. doi:10.1017/jfm.2017.350.

${ }^{13}$ Maekawa, Z., "Noise reduction by screens," Applied Acoustics, vol. 1, Jul. 1968, pp. 157-173. doi:10.1016/0003682X(68)90020-0.

${ }^{14}$ Heberling, B., "Numerical Investigation of a Shielded Chevron Nozzle," 57th AIAA Aerospace Sciences Meeting, 2019.

${ }^{15}$ Bridges, J., and Wernet, M. P., "The NASA Subsonic Jet Particle Image Velocimetry (PIV) Dataset," NASA/TM2011-023688, NASA NTRS, 2011. https://ntrs.nasa.gov/search.jsp?R=20110023688. 
${ }^{16}$ Bridges, J., and Wernet, M., "Establishing Consensus Turbulence Statistics for Hot Subsonic Jets," 16th AIAA/CEAS Aeroacoustics Conference, American Institute of Aeronautics and Astronautics, 2010. doi:10.2514/6.2010-3751.

${ }^{17}$ Wroblewski, A., Locke, R., Wernet, M., and Bridges, J., "Validating PIV Measurements in Supersonic Jets Using Shadowgraph Optical Imaging," 49th AIAA Aerospace Sciences Meeting including the New Horizons Forum and Aerospace Exposition, American Institute of Aeronautics and Astronautics, 2011. doi:10.2514/6.2011-1163.

${ }^{18}$ Bridges, J., "Effect of Heat on Space-Time Correlations in Jets," 12th AIAA/CEAS Aeroacoustics Conference (27th AIAA Aeroacoustics Conference), American Institute of Aeronautics and Astronautics, 2006. doi:10.2514/6.20062534

${ }^{19}$ Bridges, J., and Wernet, M., "Turbulence Associated with Broadband Shock Noise in Hot Jets," 14th AIAA/CEAS Aeroacoustics Conference (29th AIAA Aeroacoustics Conference), American Institute of Aeronautics and Astronautics, 2008. doi:10.2514/6.2008-2834.

${ }^{20}$ Bridges, J., and Wernet, M., "Effect of Temperature on Jet Velocity Spectra," 13th AIAA/CEAS Aeroacoustics Conference (28th AIAA Aeroacoustics Conference), American Institute of Aeronautics and Astronautics, 2007. doi:10.2514/6.2007-3628.

${ }^{21}$ Biedron, R. T. C., "FUN3D Manual: 13.3,” NASA/TM-2018-219808, 2018. https://ntrs.nasa.gov/search.jsp?R=20180001971.

${ }^{22}$ Sobachkin, A., and Dumnov, G., "Numerical Basis of CAD-Embedded CFD.," Feb. 2014. https://www.solidworks.com/sw/docs/Flow_Basis_of_CAD_Embedded_CFD_Whitepaper.pdf.

${ }^{23}$ Podboy, G. G., "Jet-Surface Interaction Test: Phased Array Noise Source Localization Results," American Society of Mechanical Engineers, 2012, pp. 381-414. doi:10.1115/GT2012-69801.

${ }^{24}$ Dougherty, R., and Podboy, G., "Improved Phased Array Imaging of a Model Jet," 15th AIAA/CEAS Aeroacoustics Conference (30th AIAA Aeroacoustics Conference), American Institute of Aeronautics and Astronautics, 2009. doi:10.2514/6.2009-3186.

${ }^{25}$ Lee, S. S., and Bridges, J., "Phased-Array Measurements of Single Flow Hot Jets," 11th AIAA/CEAS Aeroacoustics Conference, American Institute of Aeronautics and Astronautics, 2005. doi:10.2514/6.2005-2842.

${ }^{26}$ Khavaran, A., and Bridges, J., "Development of Jet Noise Power Spectral Laws Using SHJAR Data," 15th AIAA/CEAS Aeroacoustics Conference (30th AIAA Aeroacoustics Conference), American Institute of Aeronautics and Astronautics, 2009. doi:10.2514/6.2009-3378.

${ }^{27}$ Bridges, J. E., Podboy, G. G., and Brown, C. A., "Testing Installed Propulsion For Shielded Exhaust Configurations," 22nd AIAA/CEAS Aeroacoustics Conference, American Institute of Aeronautics and Astronautics, 2016. doi:10.2514/6.2016-3042.

${ }^{28}$ Henderson, B. S., and Leib, S. J., "Three-stream jet noise measurements and predictions," The Journal of the Acoustical Society of America, vol. 136, Oct. 2014, pp. 2080-2080. doi:10.1121/1.4899466.

${ }^{29}$ Bridges, J., and Brown, C., "Parametric Testing of Chevrons on Single Flow Hot Jets," 10th AIAA/CEAS Aeroacoustics Conference, American Institute of Aeronautics and Astronautics, 2004. doi:10.2514/6.2004-2824.

${ }^{30}$ Opalski, A., Wernet, M., and Bridges, J., "Chevron Nozzle Performance Characterization Using Stereoscopic DPIV," 43rd AIAA Aerospace Sciences Meeting and Exhibit, American Institute of Aeronautics and Astronautics, 2005. doi:10.2514/6.2005-444.

${ }^{31}$ Dougherty, R. P., and Podboy, G. G., "Improved Phased Array Imaging of a Model Jet," NASA/TM-2010-216227, 2010. https://ntrs.nasa.gov/search.jsp?R=20100017324.

${ }^{32}$ Engblom, W., Khavaran, A., and Bridges, J., "Numerical Prediction of Chevron Nozzle Noise Reduction Using Wind-MGBK Methodology," 10th AIAA/CEAS Aeroacoustics Conference, American Institute of Aeronautics and Astronautics, 2004. doi:10.2514/6.2004-2979.

${ }^{33}$ Khavaran, A., "Role of Anisotropy in Turbulent Mixing Noise," AIAA Journal, vol. 37, 1999, pp. 832-841. doi:10.2514/2.7531.

${ }^{34}$ Brown, C. A., "Jet-Surface Interaction Test: Far-Field Noise Results," Journal of Engineering for Gas Turbines and Power, vol. 135, Jun. 2013, pp. 071201-071201-7. doi:10.1115/1.4023605.

${ }^{35}$ Brown, C. A., and Wernet, M. P., "Jet-Surface Interaction Test: Flow Measurement Results," 20th AIAA/CEAS Aeroacoustics Conference, American Institute of Aeronautics and Astronautics, 2014. doi:10.2514/6.2014-3198.

${ }^{36}$ Brown, C. A., "Developing an Empirical Model for Jet-Surface Interaction Noise," 52nd Aerospace Sciences Meeting, American Institute of Aeronautics and Astronautics, 2014. doi:10.2514/6.2014-0878. 